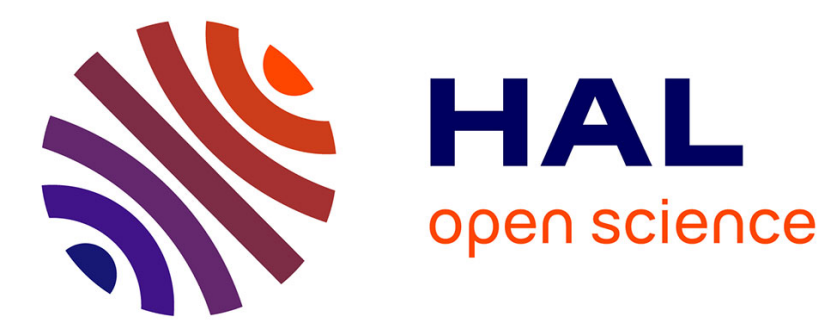

\title{
Un sanctuaire gaulois à Gournay-sur-Aronde (Oise)
}

\author{
Jean-Louis Brunaux, Patrice Méniel, André Rapin
}

\section{To cite this version:}

Jean-Louis Brunaux, Patrice Méniel, André Rapin. Un sanctuaire gaulois à Gournay-sur-Aronde (Oise). Gallia - Fouilles et monuments archéologiques en France métropolitaine, 1980, 38 (1), pp.1-25. 10.3406/galia.1980.1787 . halshs-00459004

\section{HAL Id: halshs-00459004 https://shs.hal.science/halshs-00459004}

Submitted on 4 Feb 2020

HAL is a multi-disciplinary open access archive for the deposit and dissemination of scientific research documents, whether they are published or not. The documents may come from teaching and research institutions in France or abroad, or from public or private research centers.
L'archive ouverte pluridisciplinaire HAL, est destinée au dépôt et à la diffusion de documents scientifiques de niveau recherche, publiés ou non, émanant des établissements d'enseignement et de recherche français ou étrangers, des laboratoires publics ou privés.

\section{(1) $(1) \Theta$}

Distributed under a Creative Commons Attribution - NonCommercial - NoDerivatives| 4.0 


\title{
UN SANCTUAIRE GAULOIS A GOURNAY-SUR-ARONDE (Oise)
}

\author{
par Jean-Lovis BRUNAUX, Patrice MENIEL, André RAPIN
}

\section{L.F SITF: : TOPOGRAPHIF ET STRUCTURES}

Depuis la découverte en 1975 du site du Parc à Gournay-sur-Aronde (Oise), trois campagnes de fouilles et des prospections intensives ont permis de faire considérablement avancer nos connaissances dans le domaine de la religion celte et de la typologie des armes de La Tène II. Bien que l'étude du mobilier dans sa spécificité (restauration des objets métalliques notamment) et dans son contexte archéologique (étude des relevés de fouille, stratigraphie) n'en soit finalement qu'à des débuts prometteurs et que deux campagnes de fouilles soient encore nécessaires pour dégager l'intérieur de l'enceinte cultuelle, il paraît cependant souhaitable de livrer aux archéologues une première somme d'informations. Il convient de préciser que ces dernières présentent un caractère fragmentaire et parfois hypothétique qu'on expliquera par l'état prématuré de nos recherches et par l'impossibilité de présenter ici l'ensemble des données brutes.

La principale découverte sur le site du Parc est celle d'un dépôt considérable d'ossements et d'armes celtes qu'on est en droit de rapprocher par la quantité et la datation de celui de La Tène en Suisse. Ce dépôt se trouvait dans le fossé d'un enclos qu'on peut replacer précisément dans son contexte. L'enclos, en effet, est situé au centre d'un oppidum, parmi de nombreux bâtiments gallo-romains (fig. 1).

Toutes les structures gallo-romaines et protohistoriques étaient jusqu'à présent ignorées des archéologues; elles ont cette particularité de se trouver toutes à l'intérieur de l'oppidum. Celui-ci est situé dans la vallée de l'Aronde, petite rivière très tourbeuse à la limite du plateau picard et du Noyonnais. Par sa position topographique, cet oppidum est assez exceptionnel : il appartient à la fois au type de l'éperon barré et au type de l'oppidum de méandre. Occupant, en effet, le versant sud de la vallée, il est séparé du plateau par deux grands fossés perpendiculaires, tandis que du côté de la rivière, il est protégé naturellement par des abrupts et par la rivière qui décrit à cet endroit une courbe. Néanmoins, cette dernière protection ne devait pas être suffisante; tout l'intérieur de l'enceinte, à cause de la pente du terrain, se trouvait très exposé du côté de la rivière, notamment aux projectiles. A ce défaut du système de défense, les occupants de l'oppidum 


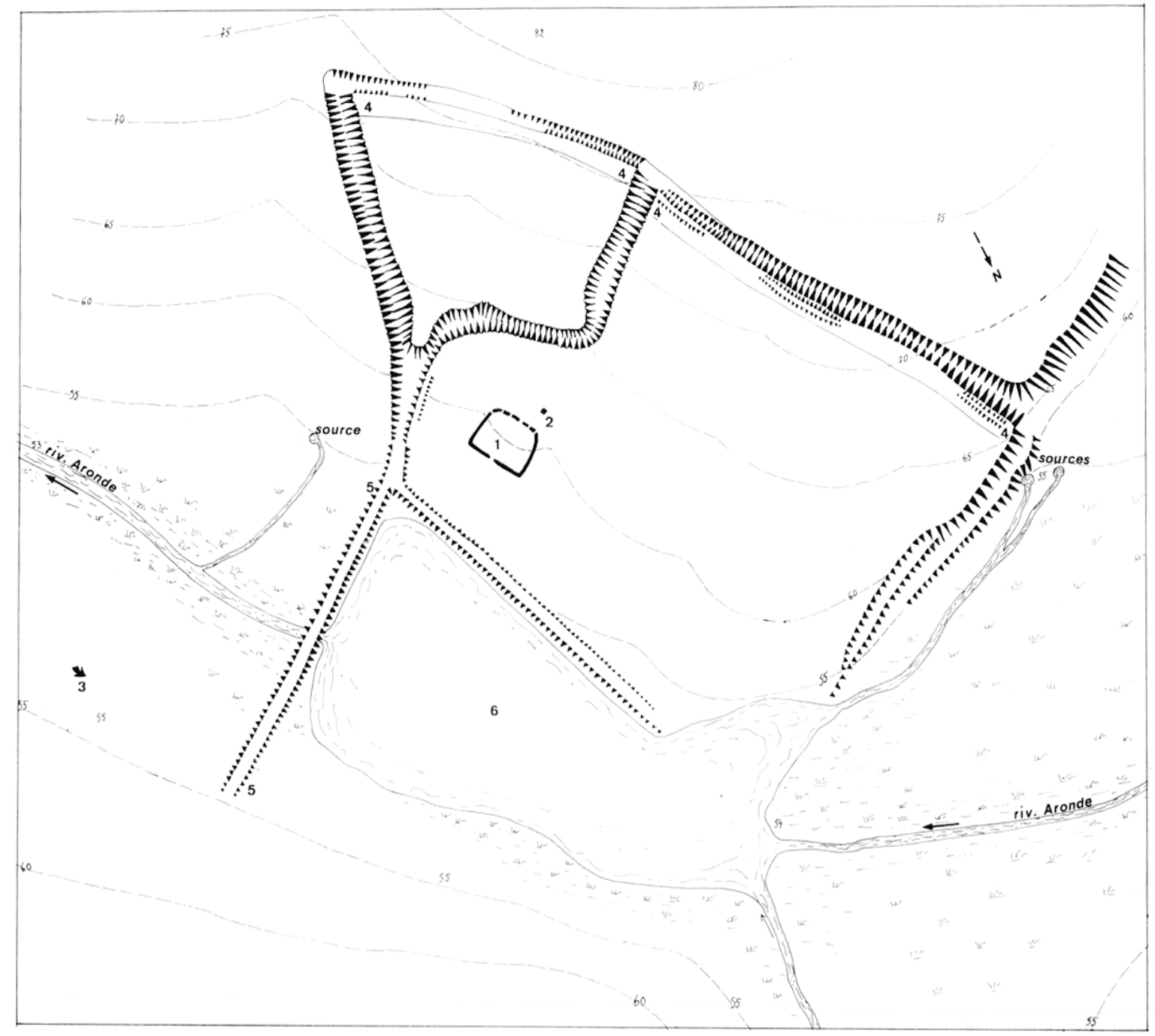

1 Plan topographique du site et de son environnement : 1, enclos cultuel ; 2 , fosse aux céramiques ; 3 , emplacement de la découverte d'un soc de charrue de La Tène; 4 , traces de Vallum ; 5 , digue; 6 , étang. (Éch.: $16 \mathrm{~mm}$ pour $100 \mathrm{~m}$ ).

trouvèrent un remède particulièrement adapté en coupant la vallée par une digue volumineuse : ils créèrent un étang qui ferait reculer les assaillants d'au moins $200 \mathrm{~m}$. Cette digue, d'autre part, permettait de franchir la vallée qui fut jusqu'au XIx ${ }^{\mathrm{e}}$ siècle une véritable barrière naturelle, en un point directement contrôlable par l'oppidum. Un tel dispositif, s'il est relativement rare, ne doit cependant pas nous étonner : les archéologues qui ont étudié les oppida du type belge et plus précisément ceux de Picardie ont à plusieurs reprises remarqué la présence de dispositifs reliant l'oppidum à la vallée et permettant un accès à la rivière ${ }^{1}$. Bien que de taille moyenne : 12 hectares, l'oppidum de Gournay vient donc renforcer l'appareil stratégique des Bellovaques qui paraît singulièrement

1 R. Agache, La déleclion aérienne de vestiges protohistoriques gallo-romains et médiévaux, numéro spécial de la Société préhistorique du Nord, Amiens, 1970, p. 37. 
sous-équipé par rapport aux autres nations belges; on remarquera enfin qu'il est situé au bord d'une zone frontière avec les Ambiens et les Viromanduens.

Dans l'angle sud-ouest de l'oppidum existe une autre enceinte dont l'espace intérieur est de 2,75 hectares. Sa forme est trapézoïdale et les fossés qui l'entourent ont une taille impressionnante : jusqu'à $20 \mathrm{~m}$ de largeur et $8 \mathrm{~m}$ de profondeur. On ne sait pas actuellement si cette enceinte est antérieure ou postérieure à celle précédemment décrite. Il est sûr en tout cas que l'ensemble du système défensif a connu plusieurs remaniements, les remparts de terre construits sur les versants très limoneux devaient être en effet particulièrement instables.

Lors de la découverte du site en 1975 , notre attention avait porté sur un ensemble de bâtiments gallo-romains que le labour avait révélé. Ceux-ci occupent l'aire centrale de l'oppidum et s'étagent du bord du plateau jusqu'aux marais. Aucun plan général de ces bâtiments n'a pu être dressé jusqu'à présent; il est donc difficile de leur attribuer une fonction : vicus, grande villa ou sanctuaire. On remarquera cependant que plusieurs bâtiments étaient construits en grand appareil, ce qui est assez rare dans les ensembles ruraux.

Abîmée également par les travaux agricoles, une fosse apparaissait parmi les structures gallo-romaines (fig. 1) ${ }^{2}$. Elle était située à quelques mètres seulement de l'angle nord-ouest de l'enclos cultuel. De forme carrée, de 2,50 m de côté, elle présentait un aspect apparemment funéraire; on découvrit à l'intérieur une vingtaine de vases rangés le long des parois selon une disposition rigoureuse, par ordre de grandeur et par type de vase. Au centre, un espace carré était vide, aucune trace d'incinération, ni d'aucun ossement humain. Par ailleurs, il semble que cette fosse très peu profonde n'ait pas été comblée immédiatement après le dépôt des vases, mais que ces derniers soient restés à l'air libre, se brisant au fur et à mesure que la fosse se remplissail naturellement. Il s'agirait donc certainement d'une fosse à offrandes que les vases nous permettent de dater du début de La Tène Ic.

\section{L'enclos culluel.}

C'est à une cinquantaine de mètres de cette fosse que furent découverts en prospection de surface quelques fragments métalliques qui révélèrent le dépôt d'armes et d'ossements de Gournay-sur-Aronde. Celui-ci affleurait le sol sur quelques mètres carrés. La fouille montra que le dépôt s'étendait en longueur et qu'il correspondait au remplissage d'un fossé délimitant un enclos (fig. 2).

Cet enclos, de forme carrée, aux angles arrondis, ne possède plus que trois côtés, le quatrième étant détruit depuis une vingtaine d'années par la construction d'une route. Le côté est, entièrement conservé, a une longueur de $45 \mathrm{~m}$ hors tout et possède en son milieu une entrée large de $3 \mathrm{~m}$ (fig. 3). Les deux autres côtés nord et sud sont incomplets. Le fossé très régulier dans son tracé varie cependant dans sa largeur et sa profondeur. C'est sur le côté est qu'il est le plus profond : 1,80 m, et le plus large : 2,50 m (fig. 3).

De toute évidence, ce côté est a été privilégié par les utilisateurs de l'enclos. C'est là

2 Informations archéologiques, Picardie, dans Gallia, 35, 1977, p. 307. 


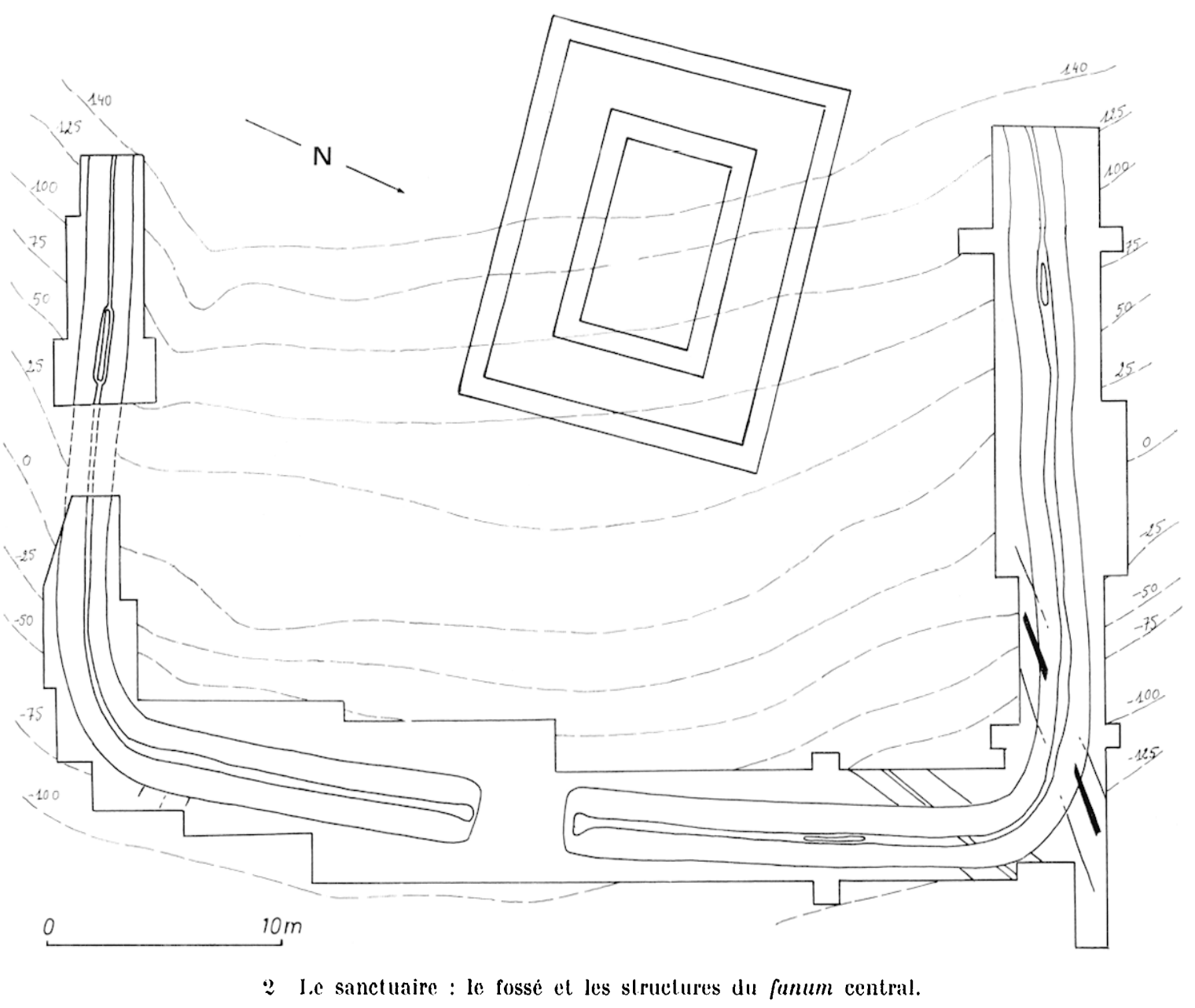

en effet qu'a été entassé le plus grand nombre d'armes et d'ossements, précisément de chaque côté de l'entrée où ils avaient été empilés les uns sur les autres pour constituer de véritables amas. C'est bien sûr le lieu de passage qu'on voulait particulièrement souligner de cette manière. A ce propos, il convient de remarquer que l'entrée est orientée à l'est mais également en direction de l'étang qui se trouvait à une centaine de mètres en contrebas.

\section{Les dépôts culluels.}

C'est en cet endroit que nous avons commencé la fouille. Il s'agissait du secteur le plus difficile à fouiller et à comprendre. De chaque côté de l'entrée, le fossé était véritablement comblé d'armes et d'os de la surface du sol jusqu'à son fond à une profondeur de $2 \mathrm{~m}$. Les armes et les os étaient inextricablement mêlés, la plupart possédait un fort pendage. Aucune lentille de terre stérile ne séparait cet amas en couches ou en dépôts. Il semblait, au contraire, que tout le matériel avait été jeté là en une scule fois. Une coupe pratiquée au centre de l'un des amas ne permettait de rien distinguer de plus.

C'est la poursuite de la fouille sur le reste du fossé qui a permis de revoir ces premières observations. En effet, de chaque côté de l'entrée, après quelques mètres, l'amas s'appau- 


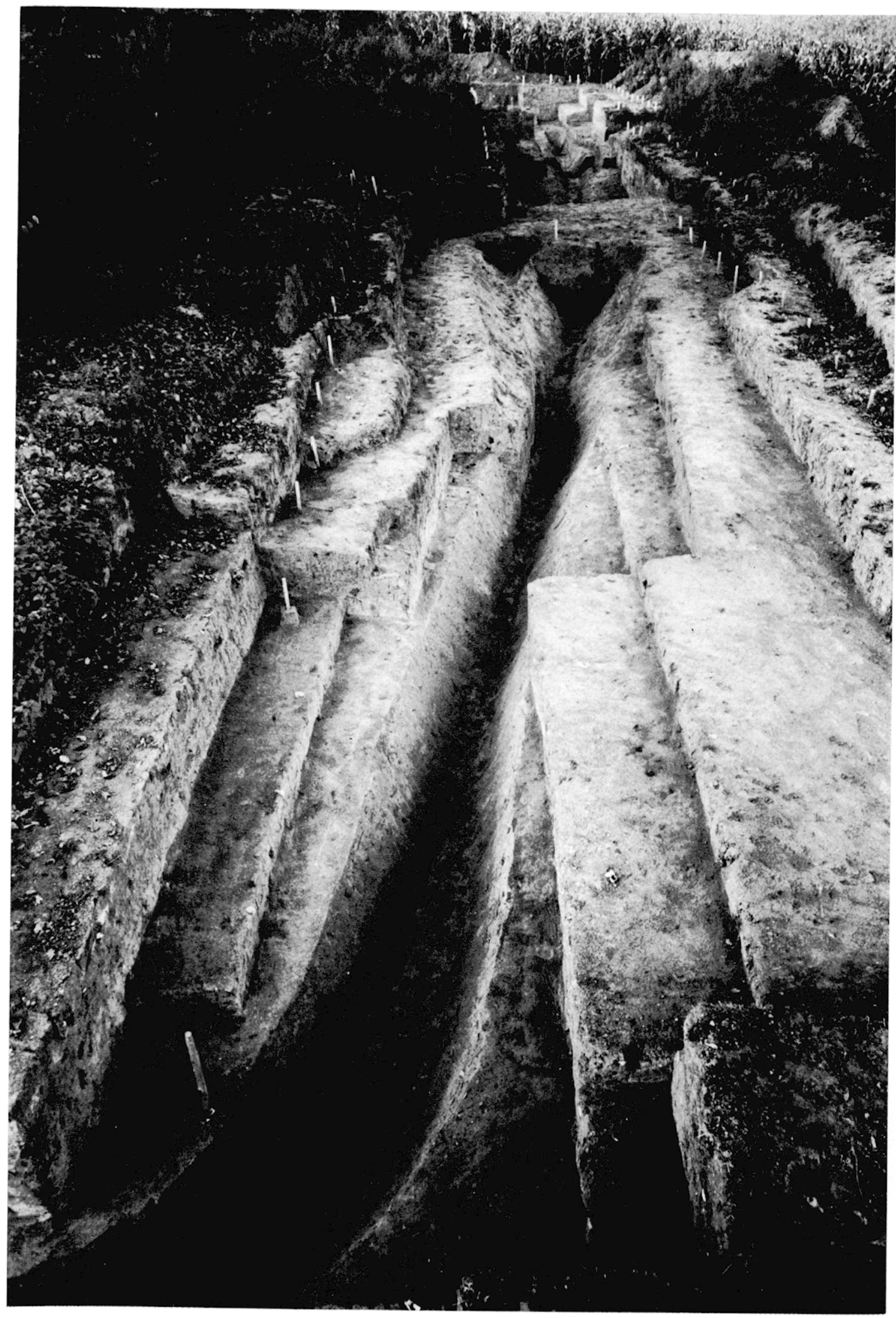

3 Le fossé : l'entrée et la façade orientale après son dégagement. 
vrissait pour se transformer en trois ou quatre couches réparties à différentes profondeurs dans le fossé et séparées par des couches de terre plus ou moins stériles et plus ou moins épaisses. D'autre part, en étudiant parallèlement à partir des relevés de fouille le matériel provenant des amas de l'entrée, et plus spécialement tous les types de liaison entre les objets (recollages, connexions, etc.), nous nous aperçumes que ces liaisons reportées sur des coupes restituées des deux amas faisaient apparaître des zones indépendantes les unes des autres, à l'intérieur desquelles les recollages se faisaient, à l'exclusion de tout recollage allant d'une zone à une autre. Cies zones étaient de véritables dépôts en forme de cuvette emboîtés les uns dans les autres et totalement invisibles lors de la fouille. Ces dépôts avaient été faits à des époques différentes, avec des périodes plus ou moins longues entre chaque dépôt.

Comment peut-on expliquer l'absence d'un remplissage naturel de terre pendant des intervalles de plusieurs années? Le fossé creusé dans le limon est très fragile et chaque hiver ses parois s'effritent, s'écroulent même, le fond se remplissant d'une couche de terre d'une épaisseur d'une dizaine de centimètres. En se penchant sur ce problème, on touche à la spécificité même de cette structure. L'étude du matériel, surtout celle des ossements dont nous reparlerons plus loin, nous apporte un certain nombre d'explications. Tout d'abord, le matériel qui était sacrifié n'était pas immédiatement apporté dans le fossé, mais seulement quelques mois plus tard, période pendant laquelle il devait être exposé. On ne le jetait pas, mais on le disposait dans le fossé suivant des principes dont nous ignorons le sens pour l'instant. Par la suite, l'ensemble du dépôt était protégé des chiens, de la végétation, du ruissellement des eaux, si bien que le dépôt suivant se faisait immédiatement sur le dernier. Mais l'aménagement du fossé de l'enclos ne devait pas s'arrêter là : ses parois étaient soigneusement protégées, peut-être par un coffrage en bois, si bien que deux siècles plus tard le tracé et la forme du fossé étaient restés les mêmes, malgré un certain nombre de modifications.

Ainsi l'étude du fossé et de sa stratigraphie nous apporte une documentation infiniment précieuse sur le rituel gaulois du sacrifice. Quelques phases essentielles de ce rituel ont déjà pu être cernées : choix des victimes et du matériel à sacrifier, différents types de sacrifice (comme on le verra plus tard dans l'étude du matériel), exposition du matériel sacrifié, dépôt, entretien des dépôts. Mais il est probable qu'en ce domaine nous n'en sommes qu'au début de nos découvertes, comme le laisse présager l'étude de l'intérieur de l'enclos.

En effet, si la fouille du fossé de l'enclos s'est révélée extrêmement positive, nous sommes en droit d'attendre que l'étude de l'espace interne de l'enclos nous apporte des documents essentiels.

Les travaux de l'été 1979 ont déjà révélé un certain nombre de structures que nous n'aborderons pas dans le détail ici : la fouille étant à peine achevée et l'étude étant en cours. Nous mentionnerons néanmoins la présence d'un fanum à l'intérieur de l'enclos dans une position légèrement décentrée (fig. 2 ). Sous ce fanum ont été trouvées des structures complexes attribuables au $\mathrm{II}^{\mathrm{e}}$ et au $\mathrm{I}^{\mathrm{er}}$ siècle avant J.-C. Il semble évident dès maintenant que la tradition du culte s'est poursuivie jusqu'à l'époque augustéenne, au moment où un 
culte véritablement gallo-romain s'est imposé sur le même emplacement. Des rapports entre les structures cultuelles gauloises de l'intérieur de l'enclos et les dépôts du fossé ont pu être établis.

\section{Jean-Louis Brunaux.}

\section{Le Mobilier mÉtallique}

L'essentiel du matériel métallique trouvé dans le fossé de l'enceinte rituelle de Gournay est constitué par des armes tant offensives que défensives. Viennent s'y ajouter seulement quelques outils et accessoires dont la fonction n'est pas toujours évidente (fig. 4).

Au total, il s'agit de plusieurs centaines d'objets en fer (le bronze étant rare), dont l'état de corrosion, ajouté à la destruction volontaire qui a précédé leur dépôt, rend difficile sinon impossible à ce jour une présentation exhaustive. Elle est en effet tributaire du travail de recherche et de restauration entrepris au Laboratoire créé à cet effet à l'Université de Technologie de Compiègne.

C'est pourquoi nous nous contenterons dans un premier temps d'une présentation simplifiée et globale de l'ensemble du matériel permettant d'évoquer les principaux problèmes liés à son analyse.

Le premier groupe de ces problèmes est de nature quantitative. Pour les raisons citées plus haut et en conséquence de la lenteur du travail de restauration, le bilan définitif du nombre des objets reste difficile à dresser actuellement. Le tableau ci-dessous est donc provisoire, approximatif, et sujet à révision au fur et à mesure de l'avancement du travail en laboratoire.

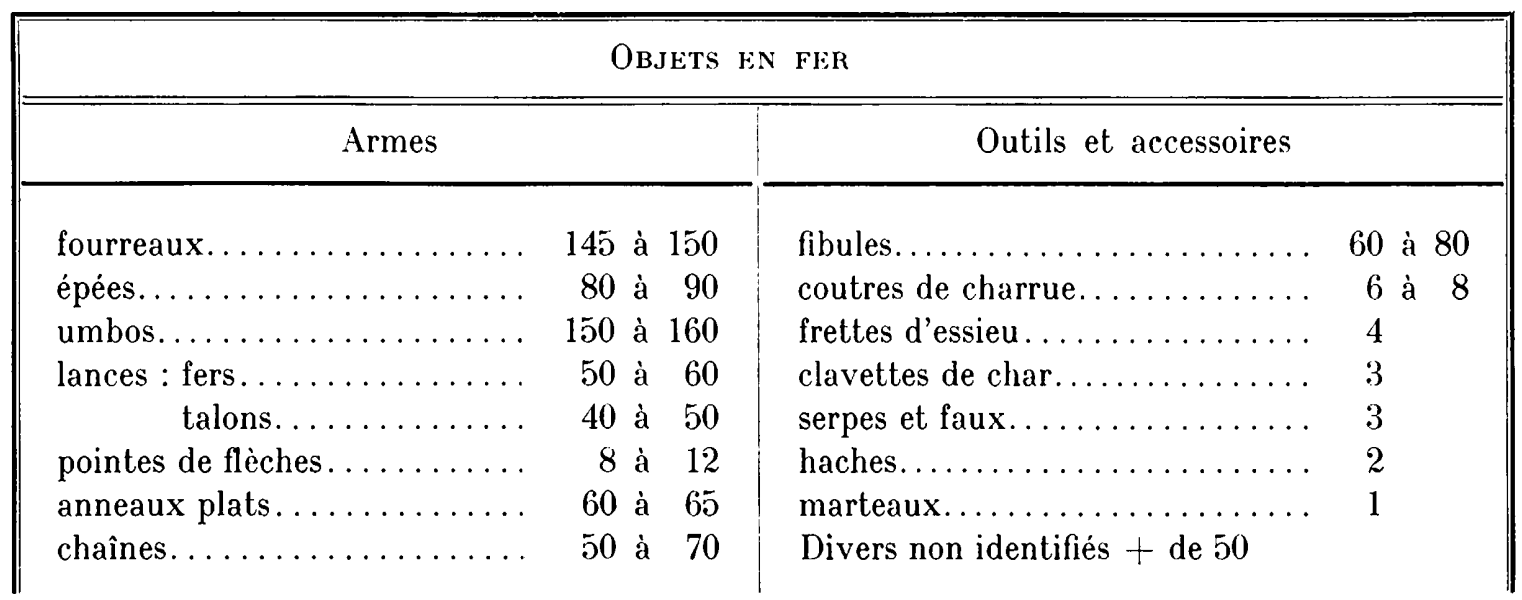

Objets en bronze et divers :

1 base d'anneau passe-guides (de joug),

1 dizaine d'anneaux plus un crochet de ceinture,

1 chaîne de suspension de fourreau (élément court),

2 fragments de bracelets plus 1 en verre,

quelques clous et rivets,

environ 600 tessons de céramique (dont 500 dans les niveaux supérieurs). 


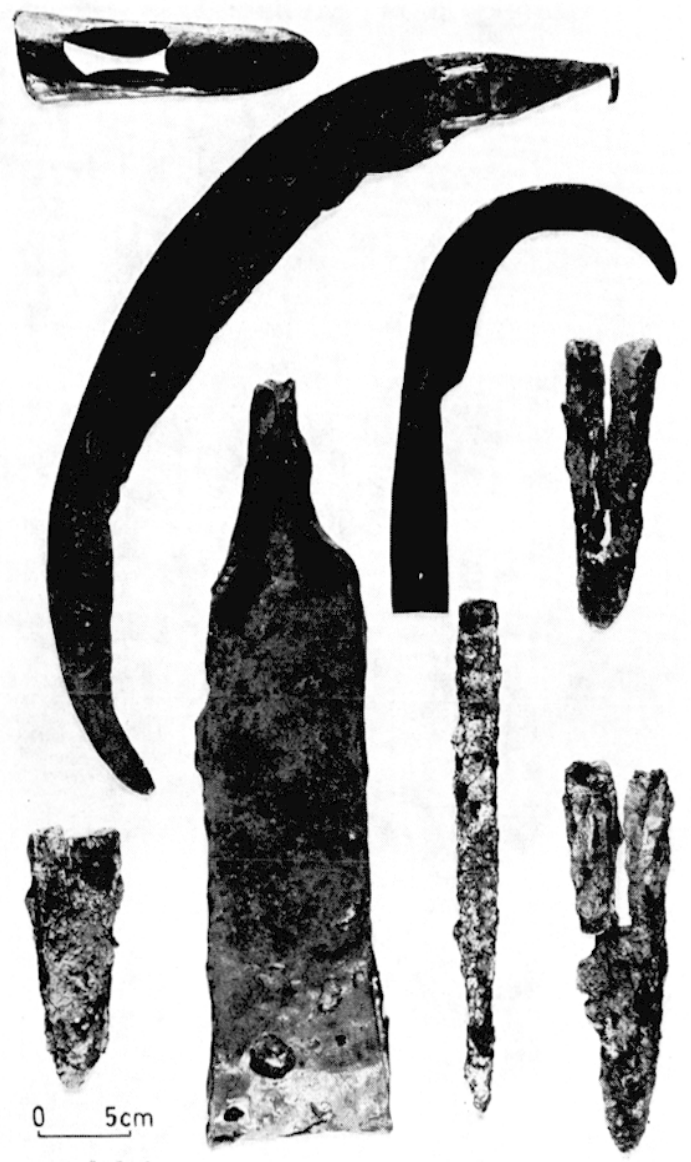

4 Outillage agricole : faux, faucille ou serpe, un grand lingot et un outil en forme de douille ajourée (en haut a gauche) dont on voit 3 intres exemplaires non restaures, probablement des coulres de charrue.

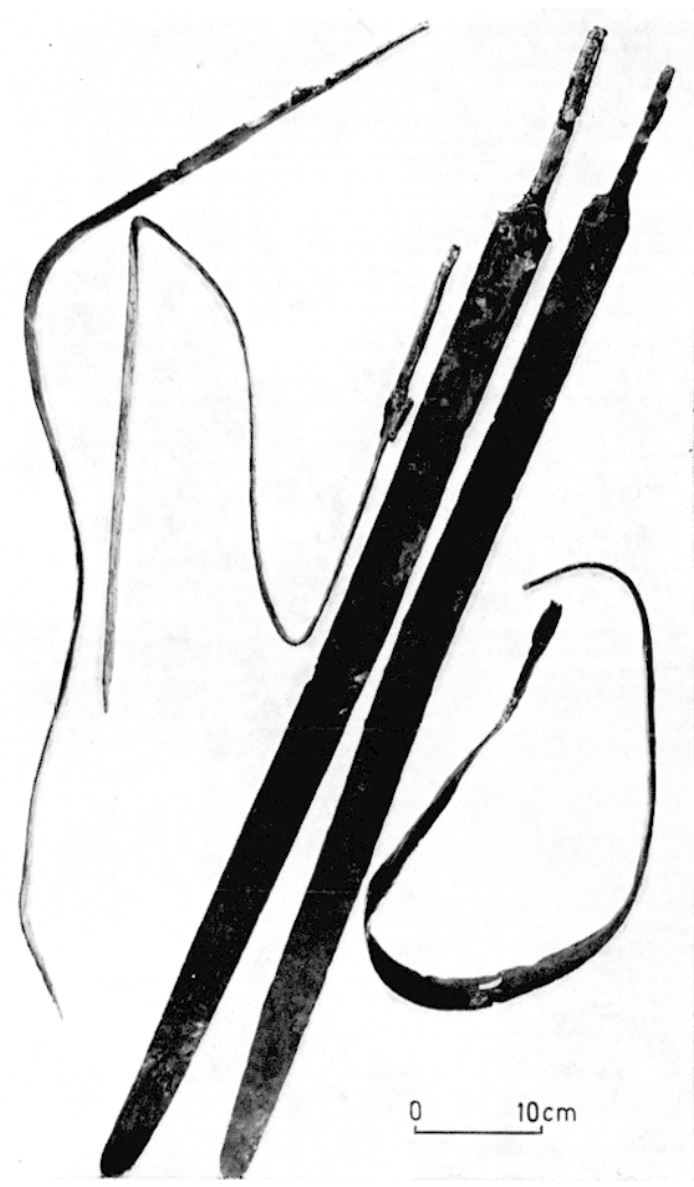

¿) Quelques épées restaurées montrant les différents types de torsions et flexions à chaud el à froid.

La simple lecture du premier tableau met en évidence quelques particularités intéressantes. Tout d'abord du côté des outils et accessoires, une domination écrasante des objets qui percent ou qui tranchent. Du côté des armes, deux grands groupes dont l'un est composé d'objets en quantité approximativement double de l'autre. A ce propos, il est curieux de constater que les épées et les fourreaux ne font pas partie du même groupe, ce qu'il serait logique d'attendre. Cette différence n'est probablement pas entièrement fortuite, d'autant qu'elle a pu être observée depuis le début de la fouille. Elle est sûrement significative, mais de quoi? Serait-elle révélatrice d'un rituel et à travers lui d'une signification symbolique du fourreau autre que celle de l'épée? I'autre part, peut-on faire un rapprochement entre le nombre des fourreaux et celui des umbos? Le fait qu'ils aient tous les deux une fonction protectrice pourrait justifier des vertus magiques analogues. Par ailleurs, on remarque que ces deux objets sont les mieux répartis sur l'ensemble du fossé et à tous les niveaux. Cette répartition dans le fossé, tant en surface qu'en profondeur, est loin d'être aussi uniforme pour le reste du matériel. Les diflérents dépôts plus ou moins étalés et très souvent imbriqués les uns dans les autres, quand ils ne sont pas, en plus, 

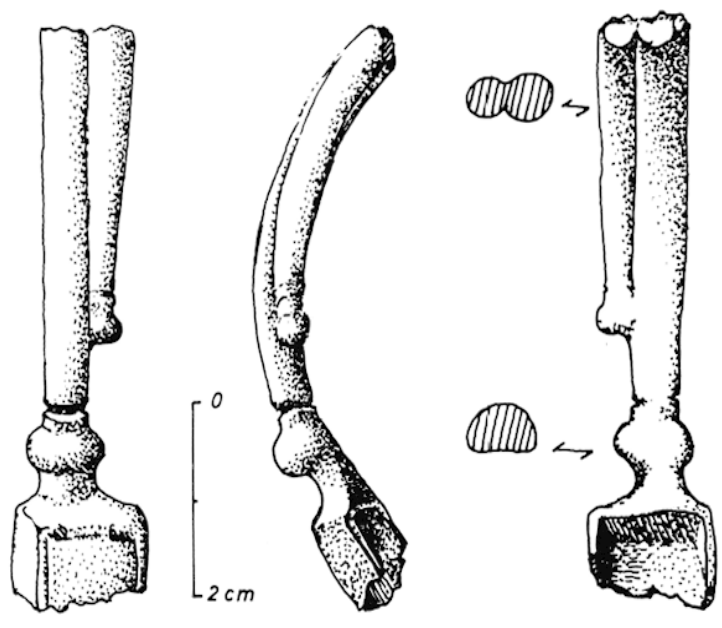

6 Fragment de bracelet en bronze

7 Fragment de bracelet en bronze à décors plastiques: $a$, vue de dessus avec la fausse soudure; b, profil et sections, hypothèse de reconstitution du bracelet; c, détails dés décors; d, développé d'un décor mettant en évidence la relation entre les plis de droite el ceux de gauche; e, systeme ornemental envisagé à partir du développé : esse enroulée autour du bracelet (évocation d'un tissu tordu ou noué) dont on aurait éliminé les aspérilés internes pour le contact avec l'avant-bras.
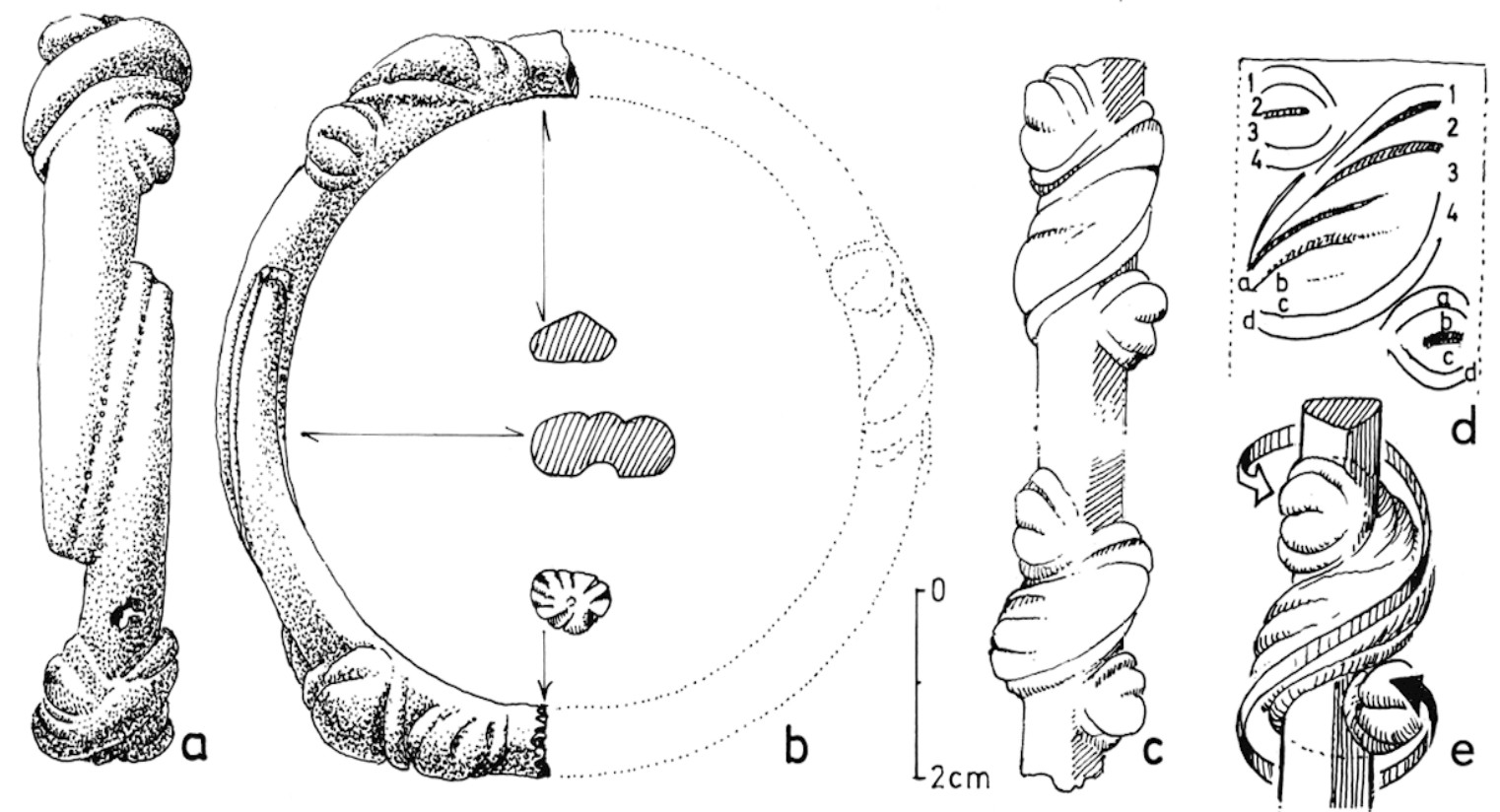

remaniés, sont très difficiles à isoler pour l'instant. La plupart d'entre eux ont été révélés par l'étude du matériel osseux. Cette stratigraphie précicuse permettra peut-être de déterminer les proportions relatives de chaque type d'objets à l'intérieur des différents ensembles.

Le dépôt le plus ancien qui a pu être ainsi isolé comprend 17 objets métalliques, dont 5 bouterolles, 2 fourreaux, 2 épées dans leur fourreau (ce qui est très rare dans les niveaux supérieurs), 3 umbos de bouclier, 1 fer de lance, une grande fibule, une chaîne de ceinture, une agrafe de fourreau et un objet non identifié.

Dans les niveaux supérieurs, nous constatons une plus grande diversification du matériel (apparition des outils), ainsi qu'une certaine spécificité. Par exemple, les anneaux plats de ceinture sont exclusivement concentrés dans les derniers niveaux, les pointes 


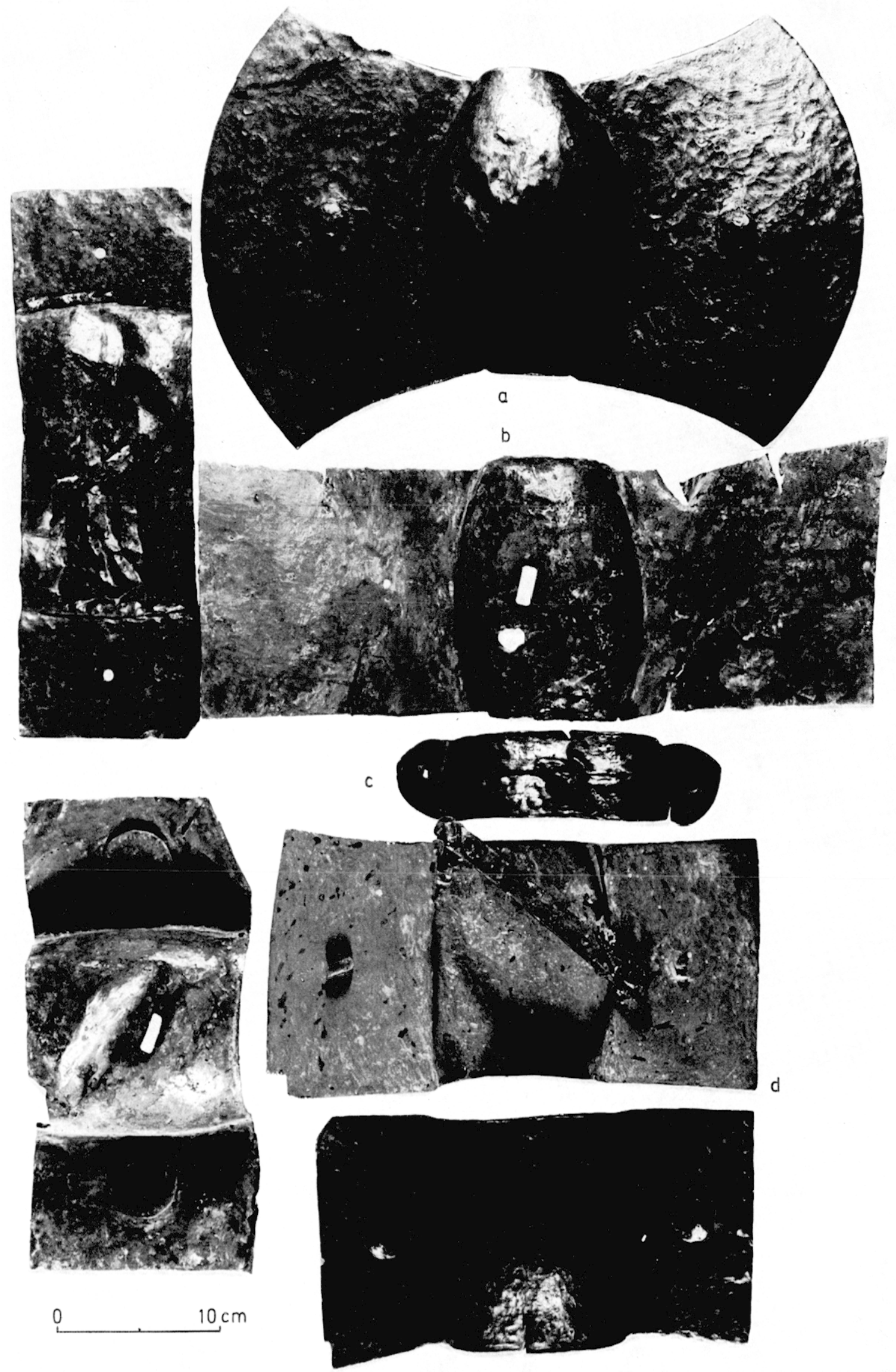

8 a, umbo à riset émaillé ; b, grand umbo à ailelles reclangulaires ; c, petit umbo à nervure médiane; d, umbo à ailettes trapézoïdales avec son manipule vue de dessous;. 
de flèches dans un niveau médian, les umbos étroits ou à ailettes trilobées et curvilignes se situent probablement tous dans un niveau inférieur (fig. 8 c).

Tous les problèmes de répartition et leurs conséquences sont donc très prometteurs quant à l'établissement d'une stratigraphic relative, constituant indispensable pour amorcer toute étude typologique cohérente.

Le type suivant de problèmes est d'ordre qualitatif. On peut les répartir en deux grands groupes. D'une part, les problèmes ayant trait à la morphologie de l'objet après sa destruction, et d'autre part, ceux liés à sa morphologie d'origine. Dans les deux cas, c'est la restauration et elle seule qui permettra d'en venir à bout. En effet, celle-ci nécessite une observation et une auscultation complète de l'objet qui dépasse de loin l'observation, pourtant de qualité, du dessinateur le plus consciencieux! Chaque restauration nécessite de nombreuses manipulations liées à des observations radiographiques minutieuses et indispensables pour guider les outils de décapage (fig. 18). Elle implique donc une compréhension intime de l'objet et de sa structure la plus profonde. En oucre, des études métallographiques sont actuellement en cours dans le département de corrosion de l'Université de Technologie de Compiègne, notamment sur les épées, les lances et certains outils, afin de préciser la nature et la composition des métaux ainsi que les technologies anciennes. Un laboratoire de restauration est donc le lieu privilégié par excellence pour mener à bien l'étude d'un tel matériel, d'où sa vocation simultanée de laboratoire de recherche permettant d'en extraire le maximum d'informations.

Ainsi la grande majorité de ces armes porte les traces d'une action destructrice variable suivant le type d'armes et les dépôts. Les épées sont parfois simplement pliées dans ou sans leur fourreau : deux types de torsion sont décelables, l'un à chaud, l'autre à froid. Certains cas de torsions spectaculaires proches du nœud n'ont pu être réalisés qu'avec un métal ponctuellement chauffé à blanc (fig. 5 et 17).

Si flexions et torsions sont plutôt spécifiques des niveaux inférieurs, les lames des épées des niveaux moyens portent souvent des traces de crantage sur leur tranchant (parfois décelables seulement sur les radiographies), de cisaillements plus ou moins profonds, voire même de martèlements. Il en va de même pour les lances, les umbos et les fourreaux dont les plaques, bouterolles et agrafes sont, en plus, souvent démontées. Dans les niveaux supérieurs, la fragmentation, ajoutée à la dispersion des morceaux, rend difficiles l'identification et la reconstitution de l'arme.

Il est certain que l'étude détaillée de ces mutilations révèle un acte précis et limité dans le temps. En regroupant des objets ayant subi des violences analogues, il sera vraisemblablement possible de préciser leur appartenance à lel ou lel dépôt el par là même d'affiner la stratigraphie relative esquissée plus haut. Il sera évidemment important de confronter cette stratigraphie avec l'étude morphologique à laquelle aboutit toute restauration (reconstitution dessinée de la morphologie originelle).

Y aura-t-il convergence ou confirmation des typologies communément admises? Certaines anomalies ou distorsions décelables dès maintenant seront-elles élucidées? Cette recherche morphologique suppose un important corollaire : l'étude stylistique des décors. Difficile à appréhender pour l'instant, elle se cantonne principalement sur les 

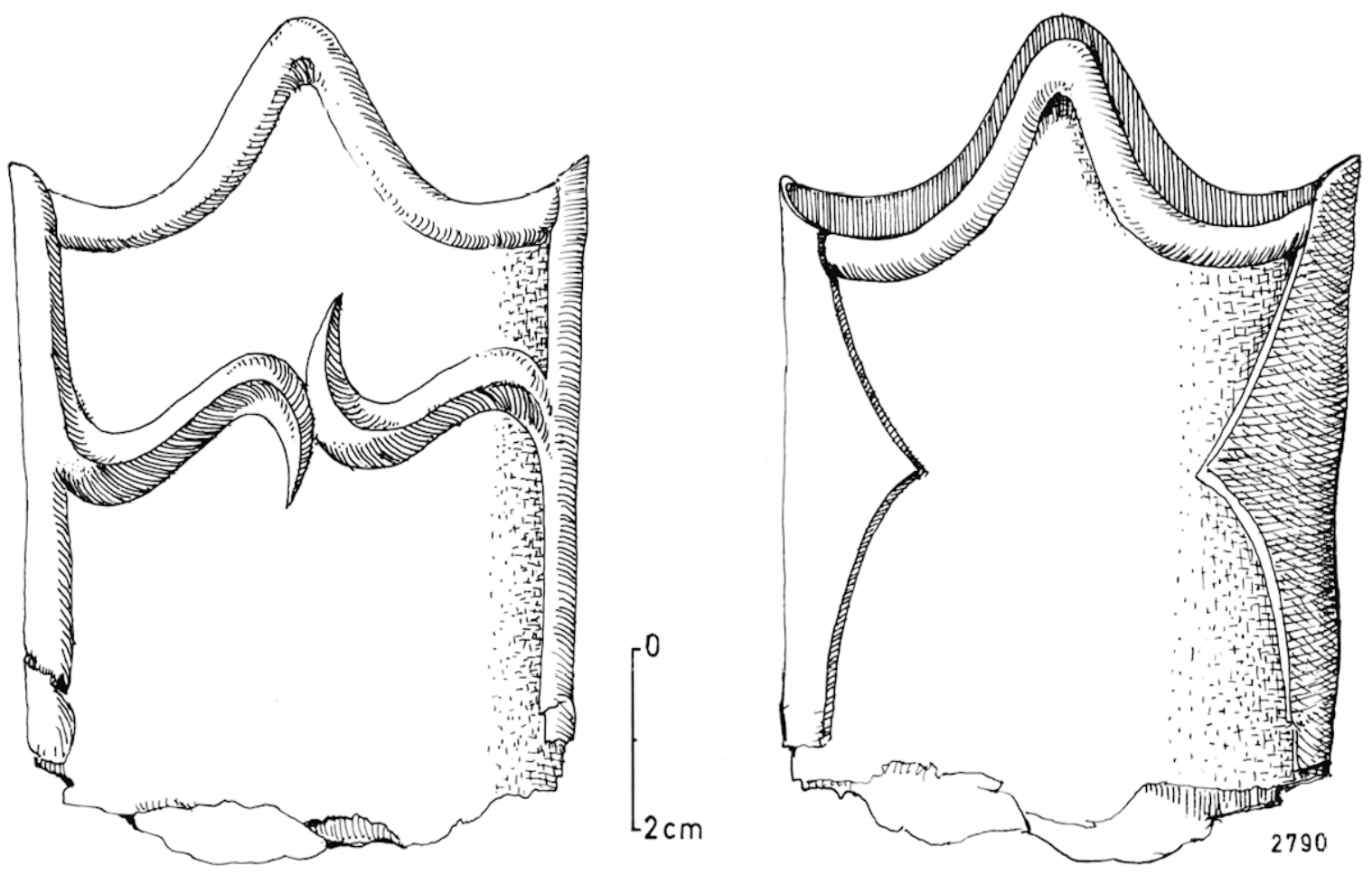

9 Entrée d'un trìs grand fourreau dont l'agrafe est composée de deux esses affrontées.

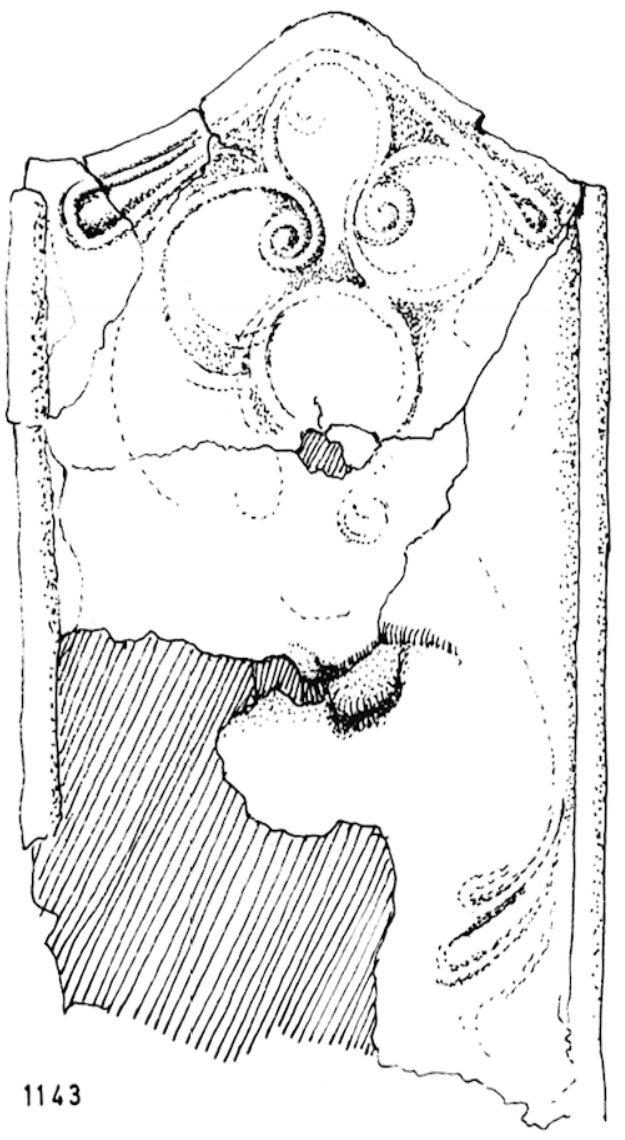

10 Entrée de fourreau à décor curviligne gravé.

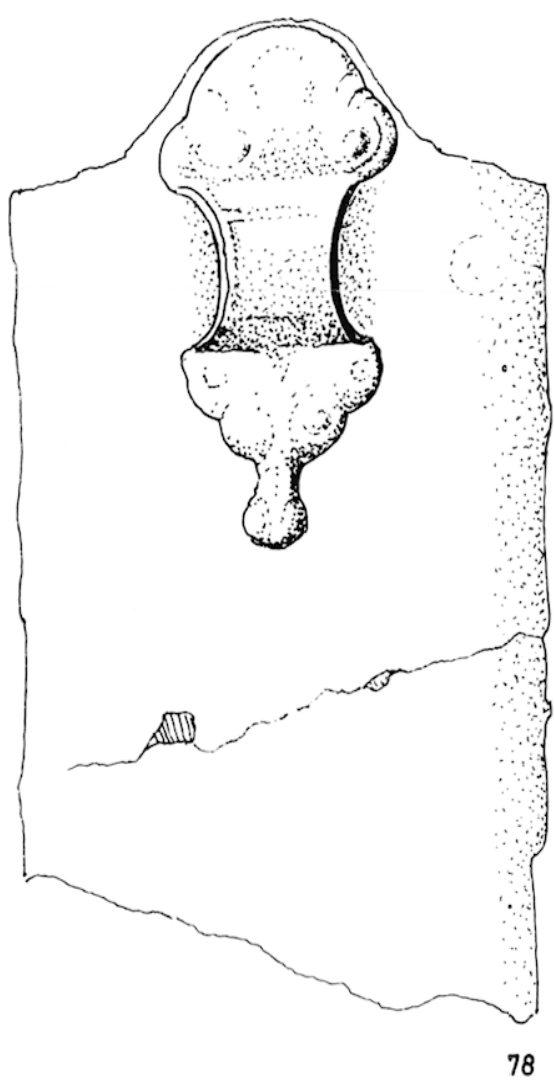

11 Piece de suspension ornementée plastiquement. La "goutte" terminant la pièce est un décor très fréquent à Gournay. 
fourreaux d'épées décorés, sur quelques umbos ainsi que sur le maigre matériel en bronze, dont les deux fragments de bracelets sur lesquels on observe des traces de fractures intentionnelles au burin (fig. 6 et 7). Le premier de ces fragments appartient à ce qu'il est convenu d'appeler "le style plastique "(fig. 7). Il est conçu comme un jonc de bronze cylindrique dont les extrémités amincies se rejoignent latéralement par une fausse soudure; les bords extérieurs de l'anneau ainsi obtenu sont rythmés par des boursouflures à caractère probablement végétal (bien que certains appellent ce genre d'ornements : escargots ou nœuds). Ce type de décor de parure annulaire est à rapprocher de ceux trouvés dans la zone danubienne, datant surtout $\mathrm{du}_{\text {III }^{\mathrm{e}}}$ siècle avant notre ère, tels ceux du musée de Teplice ${ }^{3}$.

L'autre bracelet (fig. 6), tout en présentant une analogie de structure (jonction bord à bord avec fausse soudure) est d'une conception du décor dont l'aspect est nettement plus géométrique. Il trouve son équivalent dans le tableau chronologique établi par W. Krämer pour son étude comparative du matériel de Manching, parmi les objets de la nécropole de Steinbichel, représentatifs de la Tène $\mathrm{C}$ selon la chronologie de Reinecke ${ }^{4}$. Ce style de bracelet est probablement contemporain de celui des boucles et crochets des chaînes de ceintures féminines telles celles de La Courte en Belgique ${ }^{5}$. Des affinités avec la Suisse, évidentes sur certains fourreaux, sont également décelables sur les umbos aux rivets émaillés rouge. L'un d'entre eux (fig. 8) est pratiquement identique à celui de la sépulture 26 de Vevey ${ }^{6}$, Suisse.

On observe deux types d'ornementation sur les fourreaux d'épées qui sont, comme dans tout le monde celtique, un support privilégié pour la décoration. Le premier utilise la gravure essentiellement sur la plaque avant à l'entrée du fourreau. Le deuxième utilise l'ornementation plastique des pièces fonctionnelles du fourreau, telles les agrafes, les bouterolles, les pièces de suspension (fig. 9 et 11), ainsi que des plaques rapportées ajourées. Ces deux types d'ornementation sont par ailleurs souvent utilisés simultanément sur le même fourreau. Les décors gravés empruntent le plus souvent les trois grands thèmes classiques de l'ornementation des épées celtiques.

Tout d'abord, les inévitables petits "dragons" affrontés de part et d'autre d'une nervure médiane. Le récent recensement de ce type de décor fait par J.-M. de Navarro ${ }^{7}$, s'est considérablement enrichi pour la Gaule par de nombreuses découvertes. Ce thème qui apparaît probablement au Iv ${ }^{\mathrm{e}}$ siècle dans la Celtique occidentale s'épanouit et se répand largement pendant le III $^{\mathrm{e}}$ siècle pour se maintenir en se dégradant jusqu'au $\mathrm{I}^{\mathrm{er}}$ (fig. 15).

3 Erich Lessing, Venceslas Kruta, Miklós Szabó, Les Celles, Hatier, 1978, ill., no 60 ; V. Kruta, L'art cellique en Bohême: les parures métalliques du $V^{\mathrm{e}}$ au $I^{\mathrm{e}}$ siècle avant notre ère, Paris, Champion, 1975.

4 Werner KRäMER, Manching II, dans Germania, XL, 1962, p. 306.

5 Marcel-Édouard Marien, La pér iode de La Tène en Belgique. Le groupe de la Haine, Monographie d'Archéologie nationale, 2, Bruxelles, Musées royaux d'art et d'histoire, 1961, p. 59.

6 A. NAEF, Le cimetière gallo-helvète de Vevey, dans Anzeiger für Schweizerische Alterlumstiunde, Neue Folge, III, 1901, pl. IV, p. 42-43.

7 José Maria de Navarro, The finds from the site of La Tène, II, Oxford University Press, 1972, pl. LXXIV et LXXV. Recencement complété récemment par A. BuLARD, Fourreaux ornés d'animaux fantastiques affrontés découverts en France, dans Études celtiques, XVI, 1979, p. 27-52. 

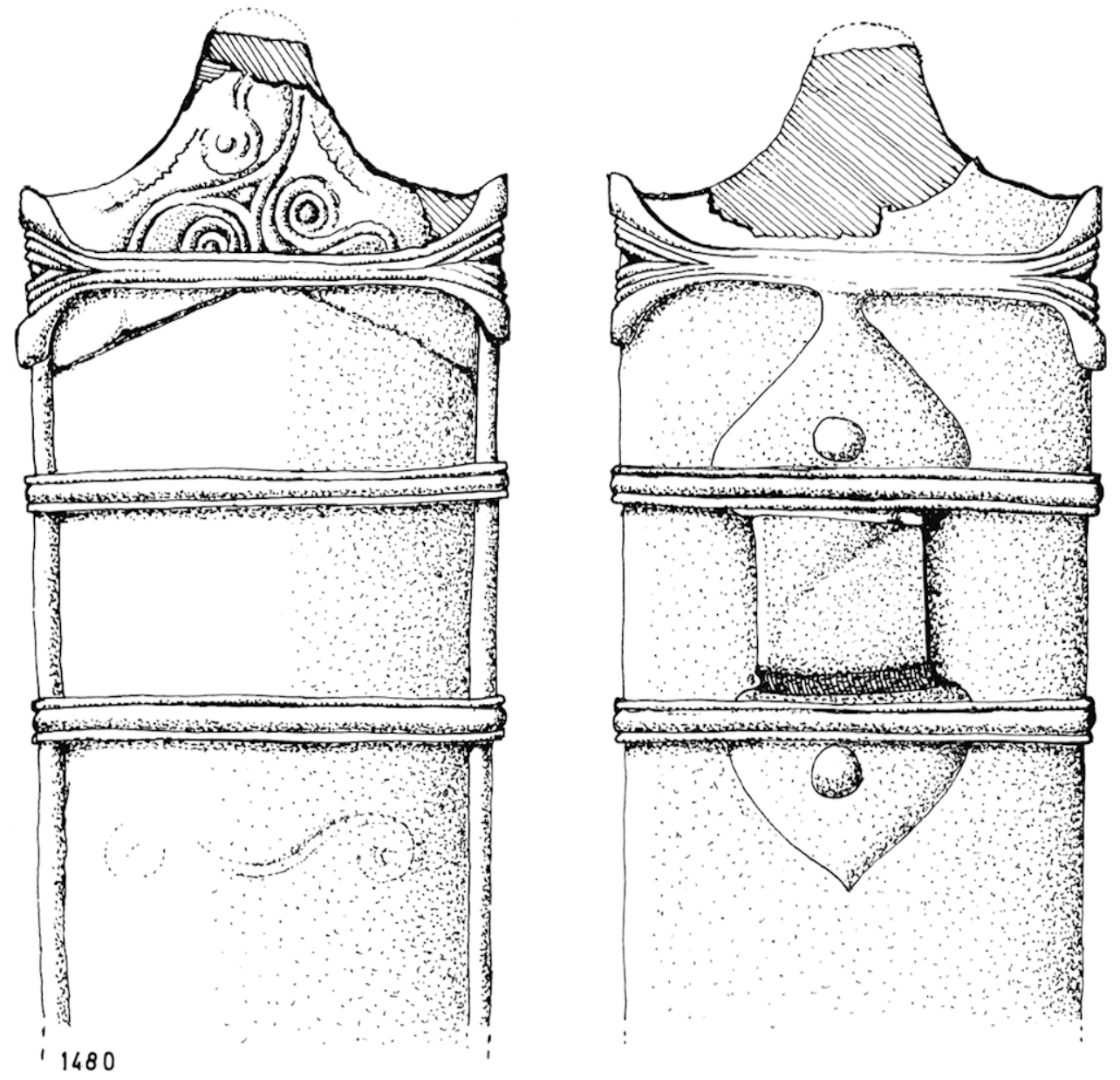

0

$2 \mathrm{~cm}$
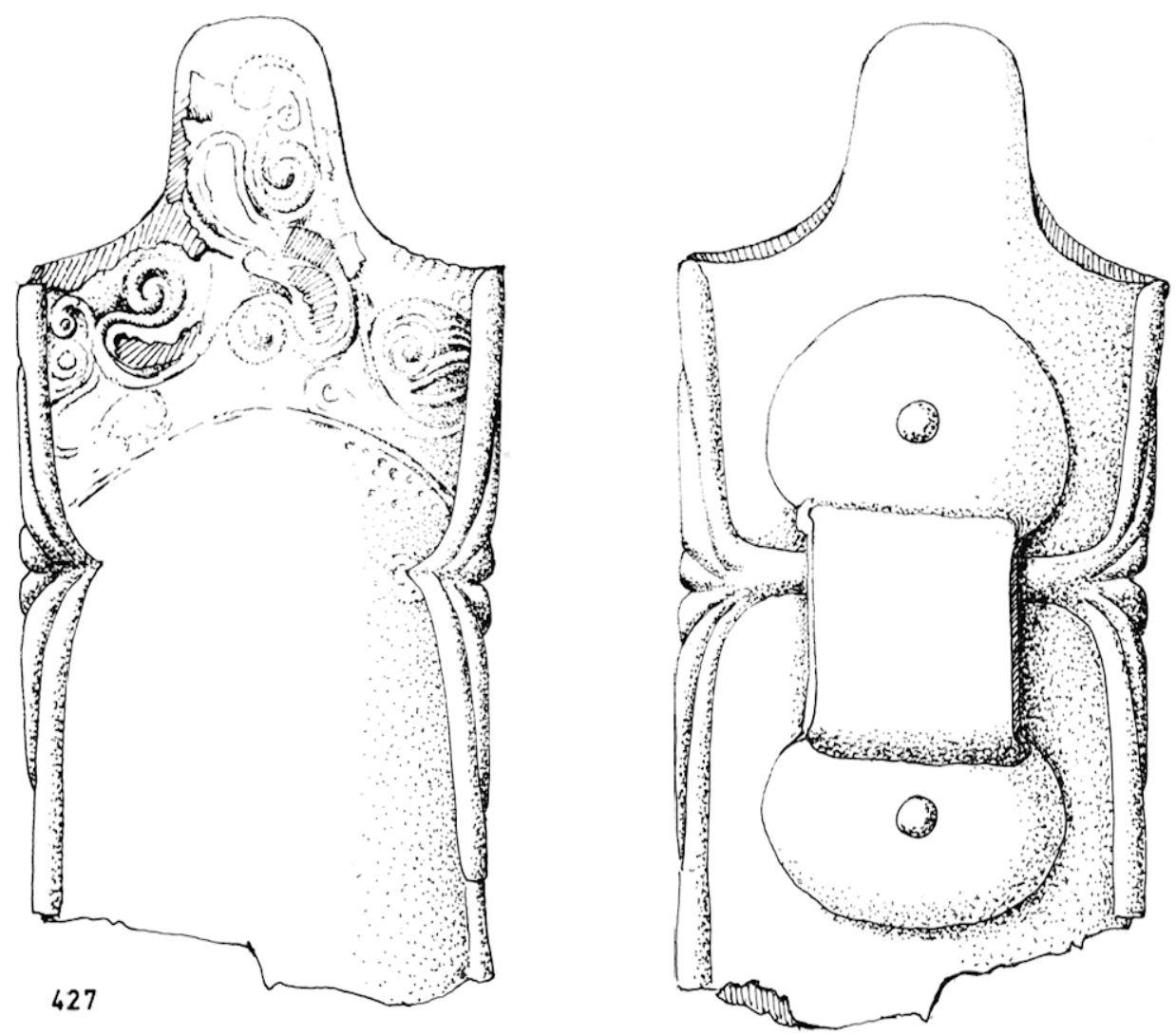

12 Entrée de fourreau avec plaque rapportée ornèe d'un triscèle gravé.

Entrée de fourreau décoré d'un triscèle prolongé de rinceaux; l'augrafo ast très richement moulurée. 

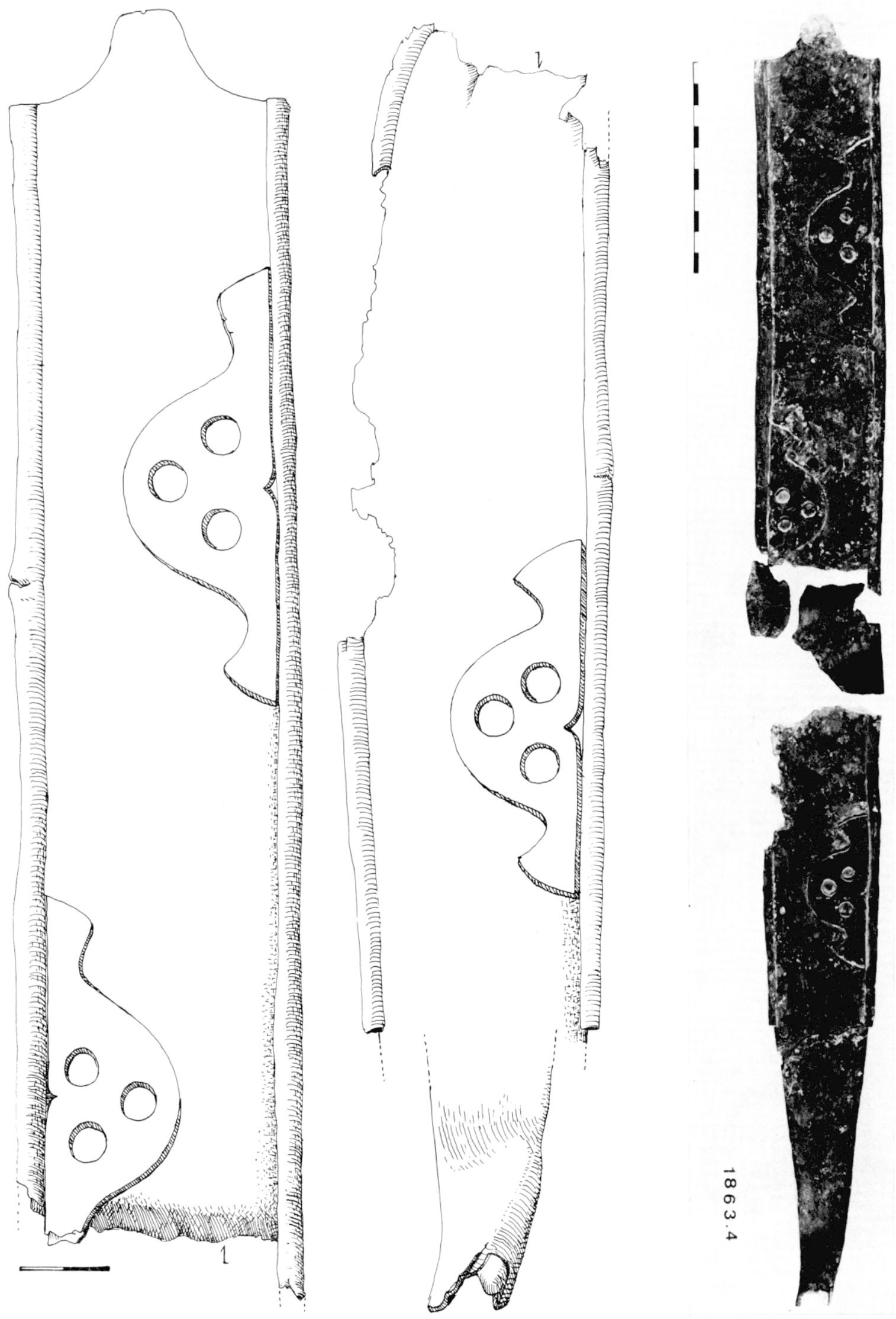

14 Plaque avant de fourreau rythmée par 3 plaques ajourées disposées en quinconce. 


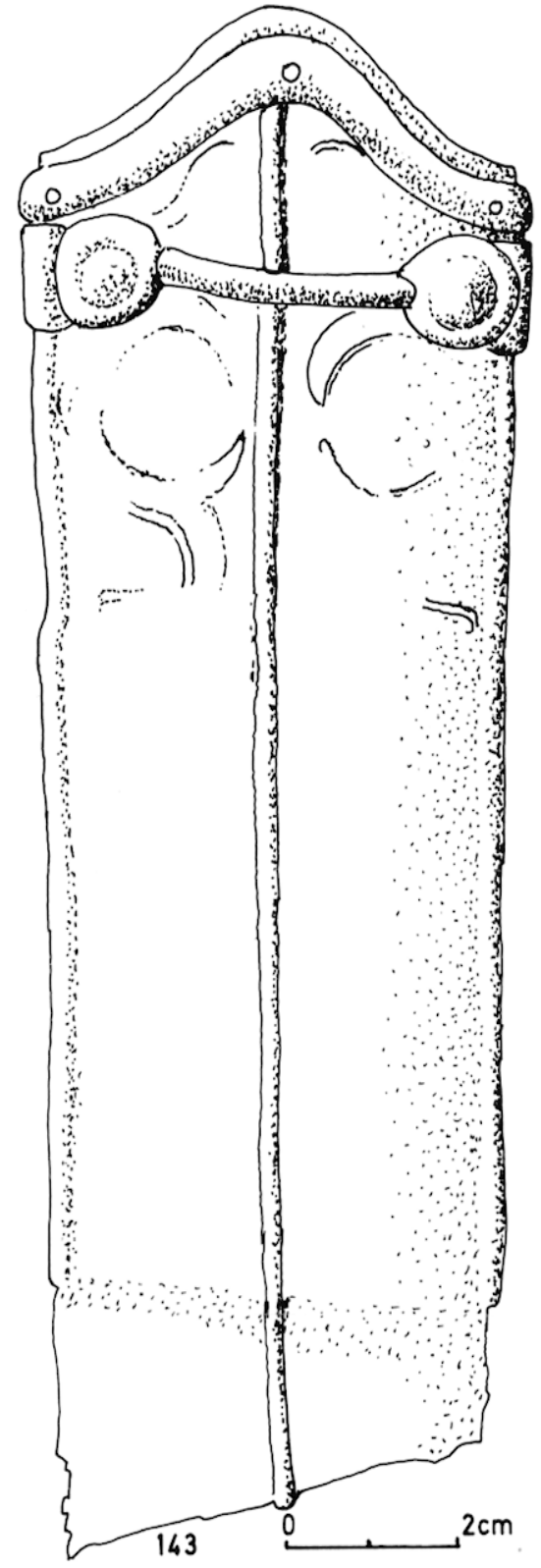

15 Fourreau à nervure médiane avec décor, malheureusement altéré, de griffons affrontés.

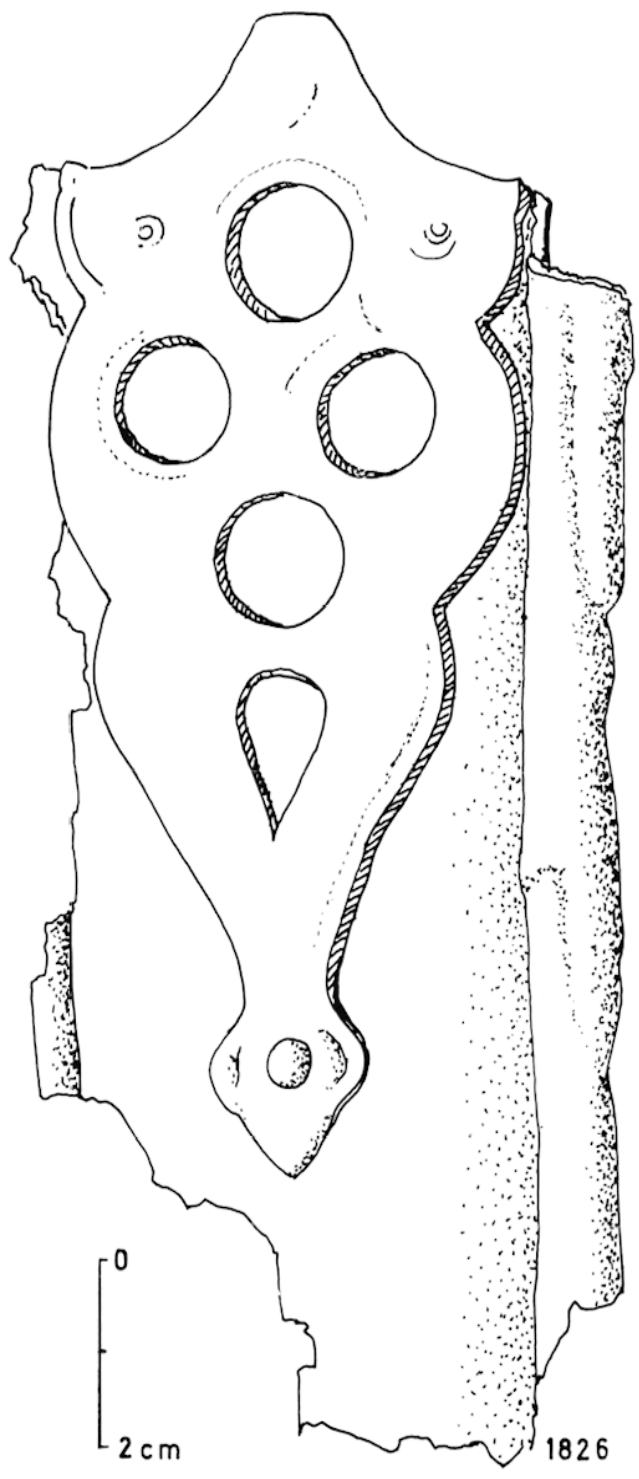

16 Entrée de fourreau décorée d'une plaque rapportée ajourée (fusion de deux griffons affrontés?).

L'autre thème dans lequel la courbe règne est apparemment plus libre dans sa conception. Après un départ presque symétrique du type pelte ou palmette, il se développe d'une manière totalement asymétrique, la plupart du temps obliquement avec rinceaux, volutes, vrilles et entrelacs. C'est ce type de décor qui constitue l'essentiel du style dit "hongrois ». L'exemplaire trouvé à Gournay (fig. 10) est à rapprocher de celui de Dobova (Yougoslavie) ou de Bodroghalom (Hongrie). 
Le troisième thème, certainement le plus répandu, surtout en territoire helvétique, utilise comme forme de base le motif du triscèle. Celui-ci, plus ou moins déformé ou prolongé par des rinceaux s'inscrivant dans la forme de l'entrée, est séparé du corps du fourreau parfois chagriné par deux obliques en forme de $\mathrm{V}$ inversé (fig. 12 et 13). Ce thème plus caractéristique du $\mathrm{II}^{\mathrm{e}}$ siècle perdure longtemps dans le $\mathrm{I}^{\mathrm{er}}$ siècle. Là encore, un exemplaire trouvé à Grournay a son équivalent à Perkáta (Hongrie) ${ }^{9}$.

En ce qui concerne les décors plastiques nous mentionnerons seulement les deux exemplaires de fourreaux à plaque rapportée et ajourée pour lesquels l'étude comparative est plus délicate. En effet, ils sont plus rares, d'une part, et d'autre part, les thèmes décoratifs utilisés peuvent appartenir aux catégories évoquées précédemment (fig. 14 et 16). Ces deux fourreaux appartiennent au même niveau, relativement ancien.

Cette étude stylistique sommaire nous a entrainés inévitablement dans des recherches comparatives indissociables des problèmes chronologiques.

\section{Problèmes chronologiques.}

Ce dernier groupe de problèmes, plus complexes, car ils doivent rendre compte de tous les autres, ne constituent plus comme souvent par le passé, la finalité essentielle de la recherche archéologique, mais simplement une étape nécessaire de celle-ci. Cela dit, on comprendra aisément que la determination de la fondation du sanctuaire de Gournay et de sa durée d'utilisation n'est pour l'instant qu'approximative et sujette, elle aussi, à révision.

Les armes (fig. 17), typologiquement les plus anciennes, appartiennent au dépôt de 17 objets cité précédemment. En effet, une des deux épées de ce lot, avec son fourreau à bouterolle ronde et ajourée, est d'un type assez proche de celle de la phase La Tène C1 de la nécropole de Münsingen (Suisse) ${ }^{10}$. Cette épée présente une analogie étroite avec celle trouvée à Saint-Maur-des-Fossés ${ }^{11}$. La ressemblance existe non seulement au niveau du fourreau mais également pour la poignée, qui a conservé sur la soie le mème nombre de rivets disposés de la même manière. De plus, une chaîne de même type, complète à Gournay, est encore fixée à sa pièce de suspension. Cette chaîne, d'une structure particulière ${ }^{12}$ révélée par la restauration (fig. 18), s'associe probablement avec une autre dont l'extrémité mâle se termine en crochet. D'autres éléments sensibles sur le plan de la chronologie, comme les deux fragments de bracelets décrits plus haut, confirmeraient une utilisation du sanctuaire dès le III ${ }^{\mathbf{e}}$ siècle avant notre ère. Par contre, les umbos de boucliers, notamment ceux à ailettes rectangulaires ou trapézoïdales, caractéristiques de La Tène moyenne, très fréquents à Gournay, sont d'une remarquable stabilité, doublée d'une longévité à toute épreuve, puisqu'on les retrouve à tous les niveaux du fossé. Ils sont donc difficilement

9 J. M. de Navarro, op. cil., pl. ClVII.

10 Frank-Roy Honson, The La Tène cemetery at Münsingen-rain, dans Acta bernensia, V, Berne, 1968, p. 29, 100,118, pl. 96,100 .

11 J. M. de Navarro, op. cil., pl. CXVI.

12 La restauration d'une chaîne identique (fig. 19) a nécessité plus de trente heures de travail à titre indicatif ; la restauration d'un fourreau décoré peut en exiger le double et parfois plus. 


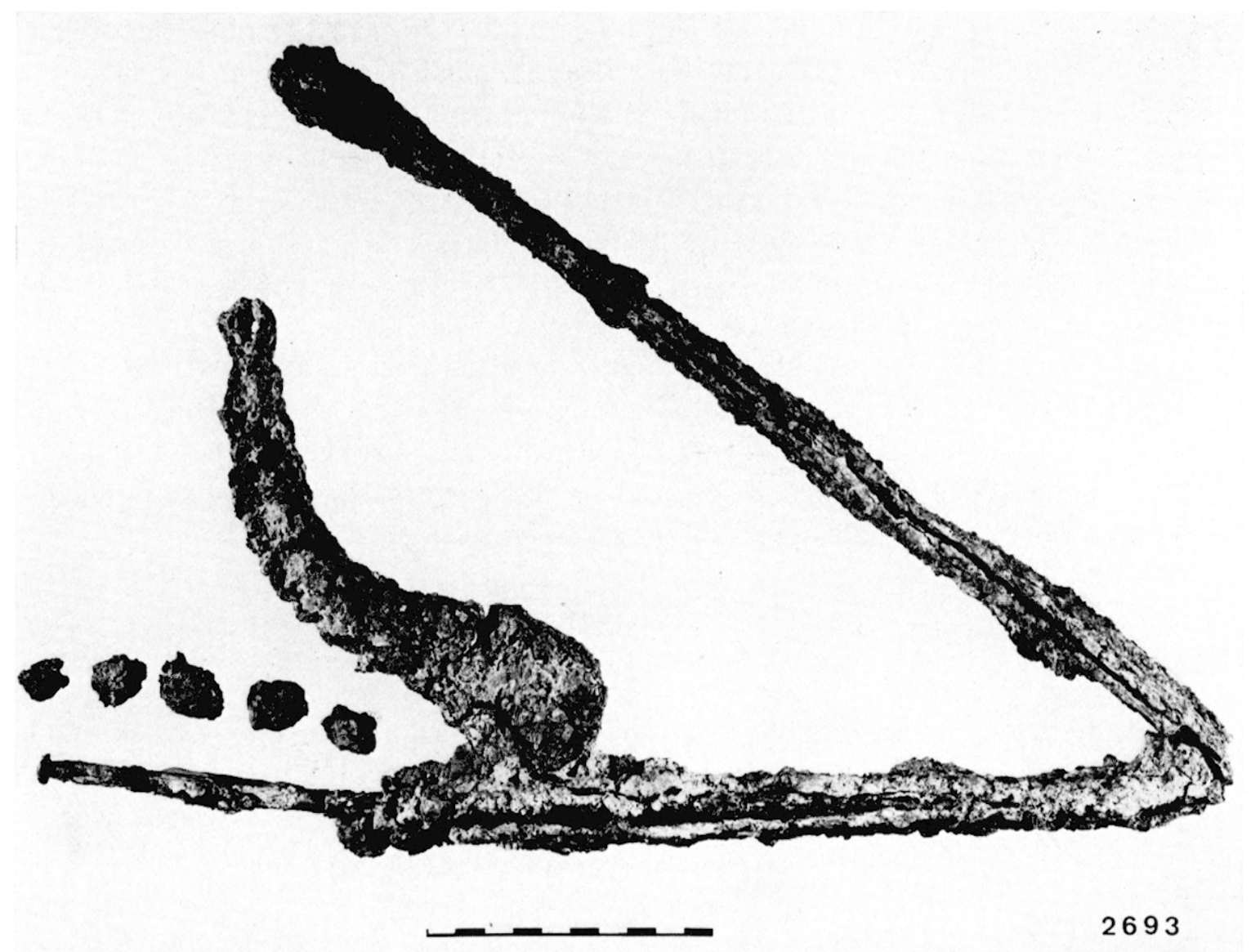

17 Épée pliée dans son fourreau avec rivels el chaîne de suspension en place.

utilisables pour l'instant comme indicateurs chronologiques. On notera également l'absence d'umbos bivalves (à deux coques), qu'on aurait pu s'attendre à rencontrer dans les niveaux les plus anciens, ainsi que celle des umbos circulaires, caractéristiques de l'époque de la conquête, tels ceux trouvés à Alésia ou sur les oppida d'Europe Centrale ${ }^{13}$. Autre absent de marque, pour nous aider à établir la limite inférieure de l'utilisation du sanctuaire : le casque. Cette absence n'est certainement pas sans significations chronologiques et culturelles.

A peine $10 \%$ des fibules en fer sont identifiables sommairement avant restauration, $\mathrm{du}$ fait de leur oxydation et surtout de leur fragmentation (une seule fibule en bronze, incomplète). On peut cependant noter qu'elles appartiennent en gros au schéma de type La Tène II, avec seulement quelques variations affectant leur taille et la forme du pied (fig. 19). L'une d'entre elles, récemment restaurée, dont la forme du pied annonce déjà la fibule du type de Nauheim ${ }^{14}$ (fig. 20), pourrait être un indice chronologique assez précis en

13 Lev Zachar, Datovanie pošiev kellskiých mecoov z Drne a Košic, dans Zbornili Stovenskèho Närodného Múzea, LXVIII, História 14, 1974, p. 71.

14 Joachim Werner, Die Nauheimer Fibel, dans Jahrbuch des Römisch-(iermanischen Zentralmuseums Mainz, 2, Mainz, 1955, p. 170-195. 

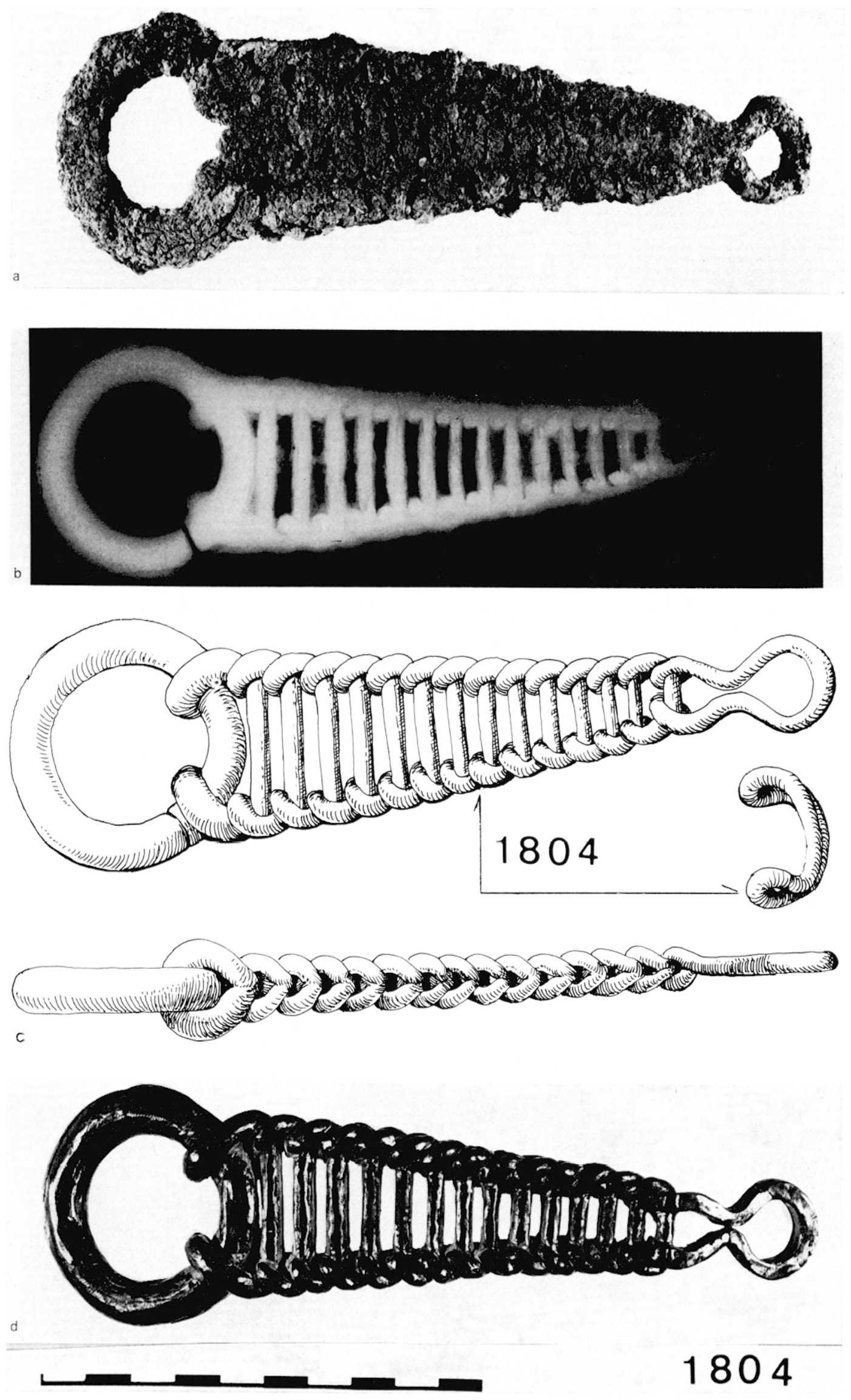

18 Différentes étapes de la restauration d'une chaîne de ceinturon : a, nettoyage; b, radiographie ; c, interprétation dessinée; d, vérification par la restauration. 


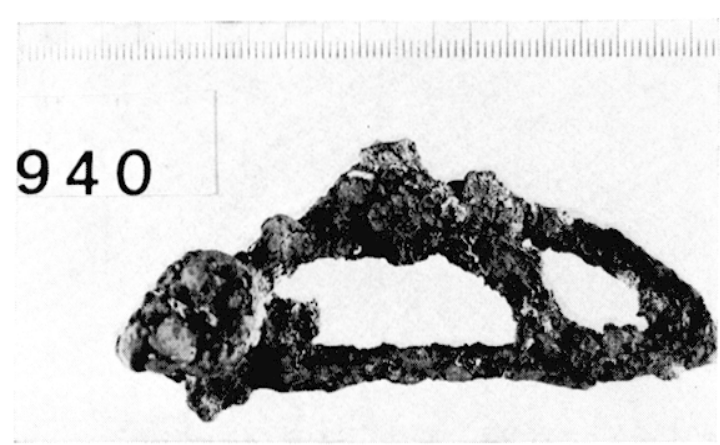

19 Fibule en fer de schéma La Tène II, type le plus fréquent à Gournay.

20 Fibule en fer de type pré-. Yauheim

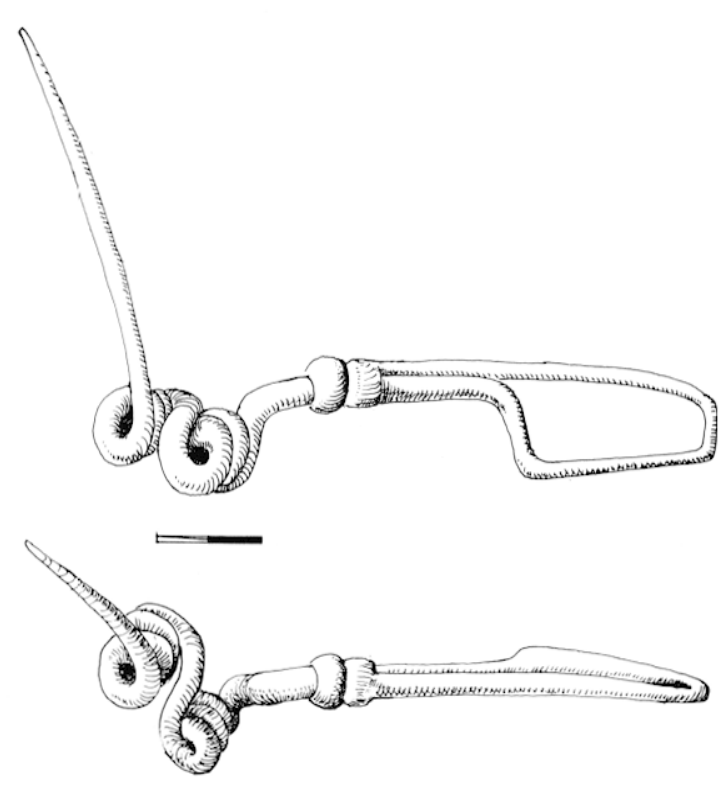

attendant la restauration de tout le lot de fibules. L'étude de celui-ci sera justement l'occasion de préciser l'évolution de la morphologie des fibules LT TII, afin de voir s'il se dégage des variations significatives sur le plan chronologique ${ }^{15}$.

Seuls les fourreaux et leurs décors, quand la restauration ou la radiographie arrive à les restituer, peuvent permettre une meilleure approche de la phase finale de Gournay. Nombre d'entre eux appartenant à des types connus de La Tène III, sont néanmoins différents de ceux décrits par Déchelette pour Alésia ${ }^{16}$ et par Zachar ${ }^{17}$ pour les oppida d'Europe Centrale. Par exemple, nous n'avons trouvé aucun fourreau à entrée droite et à nombreuses agrafes du type "fourreau à échelle ".

En définitive, toutes ces observations sembleraient indiquer l'existence d'un hiatus entre la dernière utilisation du fossé de Gournay et l'époque de la conquête, car rien ne peut être placé actuellement entre les derniers dépôts gaulois (fin du premier quart du $\mathrm{I}^{\mathrm{er}}$ siècle avant notre ère) et les niveaux augustéens qui recoupent et recouvrent la structure ${ }^{18}$.

L'intérêt essentiel du matériel de Gournay réside dans le fait que cet ensemble, exceptionnel sur le plan quantitatif, est aussi inhabituel par sa répartition dans l'espace et dans le temps, d'où son importance pour la recherche archéologique. En effet, un tel lot

15 Le même travail serail fort utile pour des objets assez mal définis chronologiquement, comme les umbos et les fers de lance.

16 J. Déchelette, Manuel d'archóologie préhistorique cellique el gallo-romaine, IV, Second àge du fer ou époque de La Tène.

17 Voir supra, n. 13.

18 Un modèle, tenant compte des données actuelles, pourrait éventuellement permeltre d'expliquer ce hiatus : en effet, si les six dépòts, identifiés par P. Meniel, sont régulièrement espacés dans le temps, ils correspondraient à des intervalles approximatifs de 25 à 30 ans (pourquoi pas un siècle gaulois : Pl.ıNs, IIist. Nal., XVI). Si l'on suppose le dernier dépôt dans le premier quart du ar s. av. notre ère, on conģoit que le suivant n'ait pu avoir lieu du fail de la conquête. 
d'armes et d'objets groupés en dépôts séparés par d'assez longs espaces de temps, constitue une source d'informations nouvelles différente de celle du mobilier des nécropoles, tant sur le plan de leur signification sociale que sur celui de l'activité religieuse des Celtes. De ce fait de nombreux points encore flous et obscurs dans le domaine de la typologie des armes et des fibules ainsi que dans celui de leur chronologie relative pourraient s'en trouver précisés.

André RAPIN ${ }^{19}$.

\section{LE MOBILIER OSSEUX}

Les ossements dégagés dans le fossé sont, dans la plupart des cas, très bien conservés et ne sont pas brisés.

Les espèces rencontrées, ainsi que les nombres de restes correspondants peuvent être résumés sur le tableau suivant :

\begin{tabular}{|c|c|c|c|}
\hline $\mathrm{B}$ & 1300 os dont 1100 intacts & Sanglier ou porc. & 210 os dont 30 intacts \\
\hline Cheval. & 230 os dont 200 intacts & Chien. & 20 os dont 4 intacts \\
\hline Ane. & 110 os dont 100 intacts & Corneille noire & $1 \mathrm{os}$ \\
\hline Mouton ou chèvre. & 550 os dont & Canard colvert. & 1 os \\
\hline
\end{tabular}

L'étude de ce matériel est en cours, et certains points nécessitent encore de nombreux approfondissements. Dans la suite on trouvera la description des observations faites sur les os par espèce, une évocation de l'approche stratigraphique et les premières hypothèses émises sur le déroulement du sacrifice.

Le bœuf : on peut considérer ces animaux comme caractéristiques à Gournay. En effet, leurs restes formaient la partie essentielle des amas de l'entrée, et ils sont de loin les plus nombreux (fig. 22). L'approche anatomique ou zoologique de ces animaux montre qu'il s'agit d'une espèce de petite taille, courante à l'époque de la Tène, comme le prouvent les comparaisons effectuées avec les données d'autres sites (en particulier celles de l'oppidum de Manching ${ }^{20}$ ) pour une grande partie de l'Europe. La taille au garrot, estimée, est de l'ordre de $1,20 \mathrm{~m}$ (fig. 25).

La présence d'une vingtaine de crânes, plus ou moins bien conservés (fig. 21), permet une détermination du sexe d'une partie de la population initiale, estimée à une quarantaine d'individus. La présence de vaches, bœufs et taureaux, dans des proportions identiques, est confirmée par l'examen des bassins.

En ce qui concerne l'âge de ces animaux, l'examen des dentitions montre un nombre restreint de jeunes individus, compensé par un grand nombre d'individus séniles. La même recherche, menée

19 Les dessins qui illustrent cet article sont le résultat d'une synthèse d'observations qui vont de celles du cliché radiographique qui sert de base (aidé parfois d'une série de tomographie) à celles, multipliées tout au long de la restauration, sur l'objet lui-même avec des éclairages d'incidences diverses. Nous avons éliminé par contre, les artefacts dus aux aléas de la corrosion (souvent représentés en pareil cas sous couvert d'une objectivité rigoureuse), qui ne font qu'alourdir inutilement le dessin, brouiller l'information au lieu de l'accrôttre et rendre difficile la lecture morphologique. Il s'agit donc, en réalité, plus d'une restitution dessinée que d'une représentation de l'objet tel qu'il apparaît actuellement, et nous nous efforçons, car là est la difficulté, de rendre cette restitution la plus objective possible. Les plans sont de M. J.-L. Brunaux et les dessins de M. A. Rapin. Les clichés sont dus à M.M. L. Lran (fig. 5, 6, 9, 18 et 20) et P. Meniel (fig. 22, 24 et 25) ; pour la fig. 19, cliché de M. L. Uran et radio. de Mile N. Caudron.

20 J. Boessneck, Die Tierknochenfunde aus dem Oppidum von Manching, Franz Steiner Verlag, Wiesbaden, 1971 . 

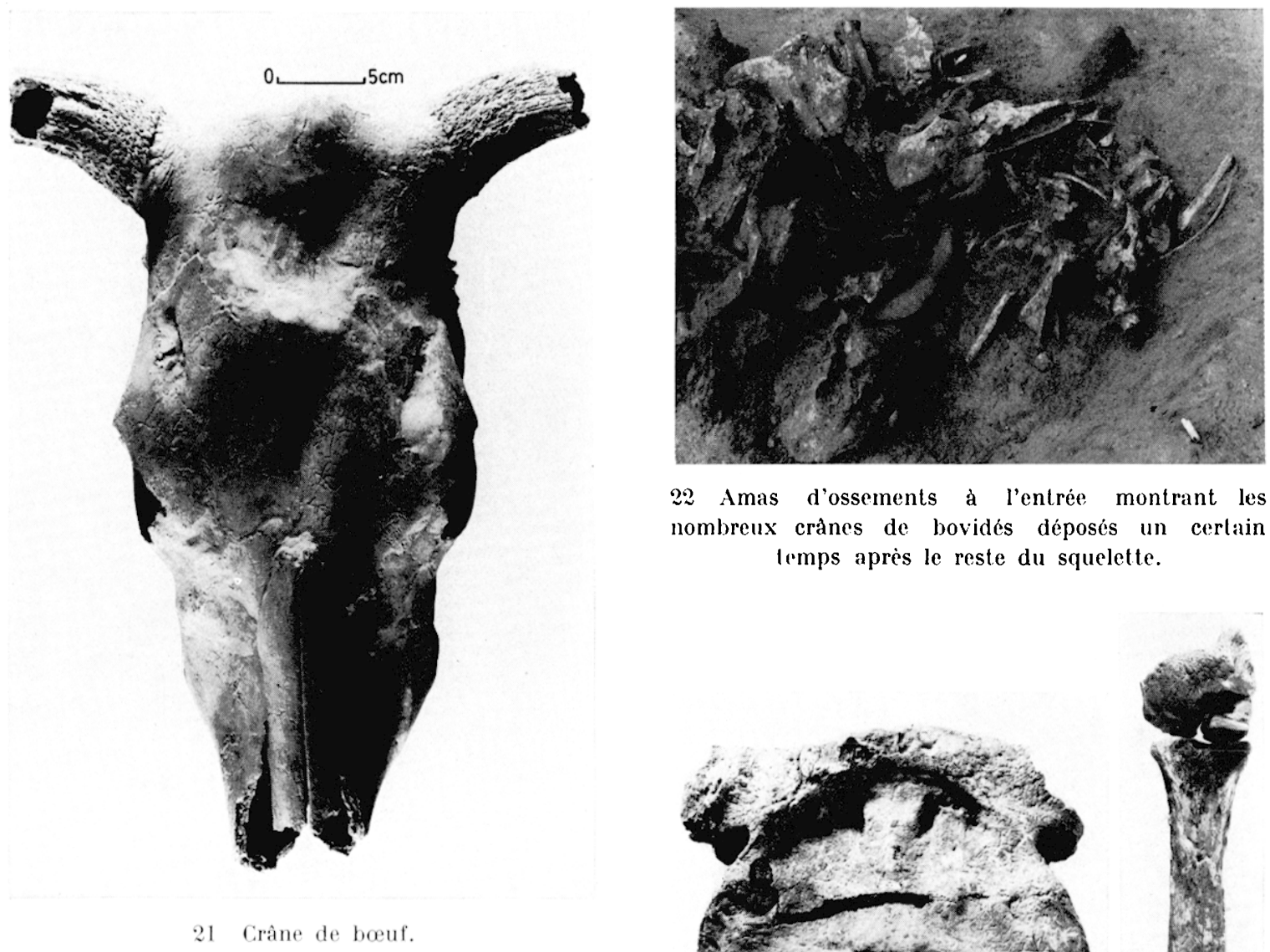

22 Amas d'ossements à l'entrée montrant les nombreux crânes de bovidés déposés un certain lemps après le reste du squelette.

23 Face nuquale d'un cràne de bouf montrant une fente due probablement à un coup de hache.
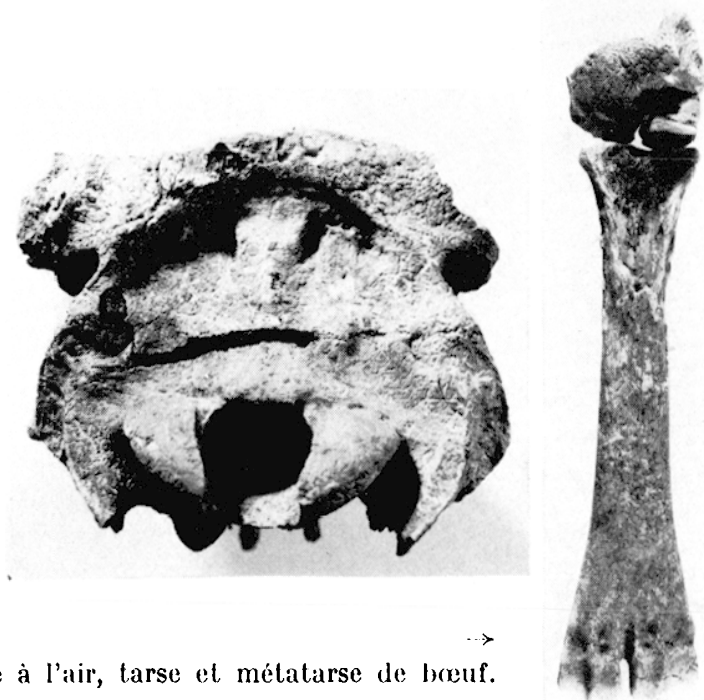

24 Os corrodé à l'air, tarse et métatarse de bouf.

à partir de l'état d'ossification des os longs . ce qui concerne une dizaine d'individus sur $40 \ldots$ montre une proportion équivalente de jeunes et d'adultes, ce qui ne change rien au résultat donné ci-dessus, sinon qu'il manque des os longs d'adultes.

Si l'on considère maintenant la composition du lot étudié, on remarque une bonne représentation du squelette axial (crâne, vertèbre el còtes, moins les queues) et une sous-représentation des os de membres et en parliculier des extrémités. Par exemple, on n'a que 14 métapodes sur les 160 que l'on aurait pu trouver, et il est improbable, vue la taille de ces os, qu'ils aient été perdus lors de la fouilie.

Certains de ces os ont été trouvés en position anatomique les uns par rapport aux autres. Ces connexions, particulièrement nombreuses pour les vertèbres -... une centaine de fragments de rachis --, sont exceptionnelles pour les membres - environ j cas ... (fig. 24). On a trouvé quelques paires de mandibules, mais tous les crânes étaient isolés.

Cette constatation nous a incités à une recherche méticuleuse des traces de découpe et de décarnation. Cette recherche s'est révélée négative. Il faut cependant signaler quelques marques douteuses sur des os longs. Par contre on a pu observer des marques trìs nettes sur les crânes, résultats de différentes opérations dont la finalité n'est pas évidente (fig. 23). 
SANGTUAIRE GAULOIS À GOURNAY-SUR-ARONDE

23

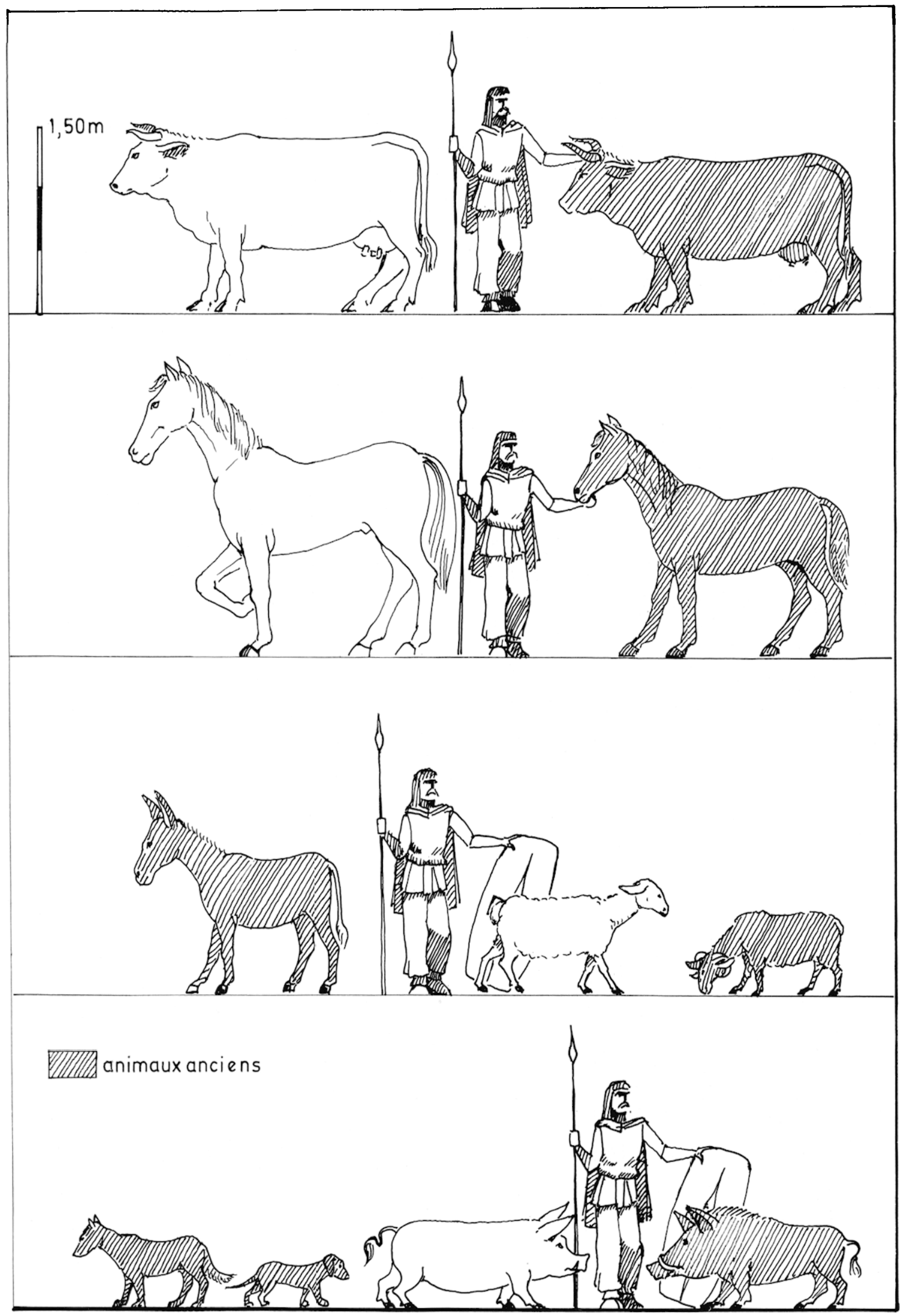

25 Taille comparée des animaux de Gournay à leurs correspondants actuels (homme : 1,70 m: La variété des races modernes d'ânes et de chiens el la diversilé de leurs tailles interdisent la comparaison. 
Le cheval et l'âne : ces animaux sont beaucoup moins nombreux que les bœufs. On compte cinq chevaux et un âne, présentant des squelettes plus ou moins complets.

L'estimation des tailles au garrot montre que les chevaux sont de taille modeste, environ 1,30 à $1,40 \mathrm{~m}$ au garrot, mais l'âne est, comparativement, de taille importante $(1,15 \mathrm{~m})$ (fig. 25$)$, sa croissance n'étant pas achevée.

Les sexes et les âges de ces animaux sont variés. En effet, l'âne n'a que deux ans et demi, alors qu'un maxillaire de cheval présente des dents totalement arasées.

Les phénomènes de conservation ont joué de façon assez nette dans certaines parties du fossé, cela rend l'étude des compositions des squelettes un peu plus délicate que pour le bœuf. Cependant, on ne note pas de phénomène aussi systématique de manque, peut-être à cause des nombres plus faibles d'os concernés. La seule caractéristique notable réside dans l'absence des humérus - un seul dégagé et qui porte des traces de décarnation - face à un nombre normal de fémurs. Les connexions anatomiques rencontrées suivent les mêmes critères que pour le bœuf (connexion au niveau du rachis et du thorax et non sur les membres).

La recherche des traces de découpe a donné lieu à deux observations : un fragment de frontal a visiblement été découpé alors que le crâne entier de jument est indemne, et un humérus d'âne porte des sillons de décarnation.

Le mouton : le nombre de restes de cette espèce est très élevé, il correspond à une centaine d'animaux au moins. Tous les os rencontrés dans les dépôts, sauf le dernier, sont des os longs de membres, les antérieurs étant très nettement majoritaires. Les autres caractéristiques sont que ces os appartiennent tous à de très jeunes individus, sauf de rares exceptions, et portent de nombreuses traces de découpe et de décarnation. L'approche des caractéristiques anatomiques de ces animaux est impossible à partir de tels éléments.

La finalité culinaire, à exclure pour les grands animaux vus auparavant, est à envisager pour cette espèce, où un morceau particulier était recherché.

Le sanglier : les restes dégagés dans le fossé peuvent provenir d'une trentaine d'individus, dont les os présentent des caractères de sanglier, cependant il peut s'agir de porc ou de sanglier domestique.

La composition du matériel est plus diversifiée que pour le mouton, ainsi que les âges - du nouveau-né à l'adulte - Quelques traces de découpe rapprochent encore ces deux espèces, dont la présence dans le fossé ne répond sans doute pas à la motivation d'exposition comme pour le bœuf.

Le chien : on ne le rencontre que dans le dépôt final, très différent des précédents, et en nombre très restreint. On peut signaler la présence d'un animal de petite taille au milieu d'une population homogène et de taille moyenne.

L'homme : les restes humains sont au nombre d'environ 70, qui peuvent provenir d'une douzaine d'individus. Ces restes sont essentiellement des os longs, dont certains portent des traces de coups, et des vertèbres cervicales, dont plusieurs portent des preuves de décapitation. Un seul crâne a été découvert mais plusieurs fragments identiques d'occiputs sont certainement riches en indices.

Données stratigraphiques : la première approche stratigraphique des dépôts d'ossements a été l'observation de lits d'ossements corrodés à l'air libre (fig. 24), pour des niveaux profonds des amas de l'entrée. La première partition obtenue a été ensuite complétée par les relations classiques que l'on peut attendre des ossements (collage, connexion, appariement, etc.); mais le schéma ainsi tracé comprenant six dépôts successifs, sera grandement amélioré par l'apport des autres catégories d'objets.

A partir de ces données, il a été possible de répartir le matéricl en lots correspondants aux différents dépôts. Il ne semble pas exister d'évolution entre des différents dépôts, 
sauf pour les quantités, jusqu'au dernier, daté de La Tène III, qui est très différent de par le remplissage, la composition et les traces relevées sur le matériel.

Le premier résultat que l'on peut attendre d'une telle approche, est une estimation de la périodicité des sacrifices, ainsi que leur stabilité, mais cela nécessite des données chronologiques que le matériel osseux ne peut apporter.

Essai d'interprétation du sacrifice : il faut d'abord faire une séparation entre les espèces consommées et celles qui ne l'ont pas été. Dans les premières on trouve le mouton, le sanglier et, plus tard, le chien, le bœuf et le cheval (dépôt T. III). Le cas du mouton est le plus frappant, car on n'a retrouvé que des restes de membres antérieurs d'agneau, et en grand nombre. Mais il n'y a pas d'indices permettant de connaître le déroulement du sacrifice de ces animaux, sinon qu'ils devaient y participer sous forme de nourriture.

Pour les bovidés et les équidés, la mise à mort, sans doute par saignée, n'était pas suivie d'une découpe - sauf peut-être pour les crânes - les os n'en portant pas de traces.

Cependant les os ayant été déconnectés avant leur mise en place dans le fossé, on peut penser à un dépôt intermédiaire - peut-être une exposition -, où les dépouilles pourrissaient jusqu'à ce que les membres se détachent, mais pas les vertèbres, ce qui laisserait supposer une durée fixe à partir du sacrifice.

Passé ce délai, les équidés étaient enfouis ou déposés en des endroits isolés du fossé, alors que les ossements de bovidé étaient entassés, en particulier aux entrées, où ils formaient des amas très visibles, exposés à l'air libre et aux intempéries pendant une période assez longue (plusieurs années).

Patrice Meniel.

Voici donc les trois apports essentiels de la découverte du Parc de Gournay-sur-Aronde. Le premier, c'est le témoignage exceptionnel qu'il nous livre sur la religion gauloise, certes témoignage brut qu'il nous faudra déchiffrer, mais qui se révèle déjà d'une étonnante richesse. Véritable document anthropologique, il devrait nous permettre d'apprécier à leur juste valeur les textes "ethnographiques" des auteurs antiques et d'aborder les sacrifices gaulois en d'autres termes que ceux de la barbarie.

Sur l'intérêt archéologique du matériel de Gournay, il n'est point trop la peine d'insister. Ce qui manquait cruellement au site de La Tène, c'est-à-dire une stratigraphie qui permette de diviser le matériel en plusieurs lots chronologiques, cette répartition en dépôts nous sommes en voie de la posséder.

Enfin le dernier apport dont nous avons peu parlé parce qu'il est prématuré, il ne devrait apparaître qu'au terme d'une synthèse : il est d'ordre historique. Les recherches archéologiques devraient nous permettre de mieux connaître le peuple gaulois des Bellovaques, peuple attesté historiquement et qui est aussi un peuple belge.

A. R. 